\title{
The phase structure of a chirally invariant lattice Higgs-Yukawa model for small and for large values of the Yukawa coupling constant
}

\author{
Philipp Gerhold \\ Institut für Physik, Humboldt-Universität zu Berlin, \\ Newtonstr. 15, D-12489 Berlin, Germany \\ E-mail: gerhold@physik.hu-berlin.de
}

\section{Karl Jansen}

$D E S Y$,

Platanenallee 6, D-15738 Zeuthen, Germany

E-mail: Karl.Jansen@desy.de

ABSTRACT: We consider a chirally invariant lattice Higgs-Yukawa model based on the Neuberger overlap operator $\mathcal{D}^{(o v)}$. As a first step towards the eventual determination of Higgs mass bounds we study the phase diagram of the model analytically in the large $N_{f}$ limit. We present an expression for the effective potential at tree-level in the regime of small Yukawa and quartic coupling constants and determine the order of the phase transitions. In the case of strong Yukawa couplings the model effectively becomes an $O(4)$-symmetric nonlinear $\sigma$-model for all values of the quartic coupling constant. This leads to the existence of a symmetric phase also in the regime of large values of the Yukawa coupling constant. On finite and small lattices, however, strong finite volume effects prevent the expectation value of the Higgs field from vanishing thus obscuring the existence of the symmetric phase at strong Yukawa couplings.

Keywords: 1/N Expansion, Lattice Gauge Field Theories, Higgs Physics. 


\section{Contents}

1. Introduction 1

2. The model 2

3. Large $N_{f}$-limit for small Yukawa coupling parameters 6

4. Large $N_{f}$-limit for large Yukawa coupling parameters 10

5. Summary and conclusions 22

A. Details of calculation I 23

B. Details of calculation II 24

\section{Introduction}

Non-perturbative investigations of lattice regularized Higgs-Yukawa models as a limit of the electroweak sector of the Standard Model have been subject of many investigations in the early 1990's, see e.g. the review articles of refs. [1 - 1 . These lattice studies were motivated by the interest in a better understanding of the fermion mass generation via the Higgs mechanism on a non-perturbative level. In addition, the focus has been on the determination of bounds on the Higgs mass and the Yukawa couplings which translate directly into bounds on the - at that time not yet discovered - top quark mass. However, these investigations were blocked, since the influence of unwanted fermion doublers could not successfully be suppressed. Moreover, the lattice models of these studies suffered from the lack of chiral symmetry. The latter, however, would be indispensable for a consistent lattice regularization of chiral gauge theories such as the Standard Model of electroweak interactions.

Here, we want to extend these earlier investigations in a new direction in order to overcome the previously encountered drawbacks by following the proposition of Lüscher [\$] for a chirally invariant lattice Higgs-Yukawa model based on the Neuberger overlap operator [9]. Within this model an exact lattice chiral symmetry can be established while suppressing the fermion doublers at the same time. This is possible despite of the Nielsen-Ninomiya theorem [10], since the established lattice chiral symmetry is not the continuum chiral symmetry itself, but recovers the latter symmetry only in the continuum limit. We consider here a Higgs-Yukawa model including only the two heaviest fermions, i.e. the top-bottom doublet, and the Higgs fields. This simplification is reasonable, since the fermion-Higgs coupling is proportional to the fermion mass and hence small for the light doublets. We 
also neglect any gauge fields within this model, since they can be taken into account via perturbation theory.

As a first step towards a numerical investigation of this Higgs-Yukawa model we begin by studying its phase structure. Here we present an analytical investigation of the phase diagram in the large $N_{f}$-limit following refs. [11, 12]. We refer the reader to these references for earlier works on lattice Higgs-Yukawa models. (See also ref. 13] for a first account of our work.) In the present paper we access the phase structure of the model at small and at large values of the Yukawa coupling constant, putting particular emphasis on the existence of a symmetric phase also in the strong Yukawa coupling regime. The latter strong coupling regime of a closely related, chirally invariant Higgs-Yukawa model in two dimensions was also studied in the recent work [14] and corresponding Monte-Carlo simulations, performed in that model, support the existence of such phase [15]. Extensions of our present paper, in particular addressing the question of lower and upper bounds on the Higgs boson mass, will be discussed in forthcoming publications.

We remark here that for the latter reasons we are eventually interested in the physical setting $N_{f}=1$. Although the large $N_{f}$ analysis is not supposed to match the $N_{f}=1$ case well on a quantitative level, we expect the large $N_{f}$ phase diagram to have the same qualitative structure. Thus, it can provide an useful orientation also for the case of $N_{f}=1$. The results from the large $N_{f}$ analysis performed here have been confronted with numerical simulations in ref. [16] and indeed, a good qualitative agreement has been found at small values of $N_{f}$.

The outline of this paper is as follows: In section 2 we briefly describe the HiggsYukawa model considered here. In the following section 3 we derive an expression for the effective potential in terms of the amplitudes of the constant and staggered modes of the Higgs field, which is a reasonable approximation at small values of the Yukawa and quartic coupling constants. We then present the resulting phase diagram in the large $N_{f}$-limit and determine the order of the occurring phase transitions. The phase structure in the regime of large values of the Yukawa coupling constant and arbitrary quartic coupling constants is then accessed by means of a different large $N_{f}$-limit presented in section 4 . We show that a symmetric phase also exists at large Yukawa coupling constants. On small lattices, however, this symmetric phase is shadowed by finite volume effects preventing the expectation value of the Higgs field from vanishing. We then end with a short summary and outlook.

\section{The model}

Aspiring to investigate the Higgs Sector of the Standard Model of electroweak interactions, we consider here a four-dimensional, chirally invariant lattice Higgs-Yukawa model containing one four-component, real Higgs field $\Phi$ and a number of $N_{f}$ fermion doublets. The latter are represented by eight-component spinors $\psi^{(i)}, \bar{\psi}^{(i)}$ with $i=1, \ldots, N_{f}$. However, these $N_{f}$ doublets are all degenerated within this model and correspond to the heaviest fermion doublet only, i.e. to the top-bottom doublet. This is a reasonable simplification due to the fermion-Higgs coupling being proportional to the fermion mass. We have introduced 
the fermion doublet number $N_{f}$ nonetheless, because it will be possible to access the model analytically in the limit of large numbers of (degenerated) fermion doublets.

Furthermore, there are also $N_{f}$ auxiliary fermionic doublets $\chi^{(i)}, \bar{\chi}^{(i)}$ present in the model, which serve as a construction tool for the creation of a chirally invariant Yukawa interaction term. However, once the chiral invariance is established, these unphysical fields can be integrated out leading to a more complicated model depending then only on the Higgs field $\Phi$ and the $N_{f}$ physical fermion doublets $\psi^{(i)}$. The partition function of the given model can be written as

$$
Z=\int D \Phi \prod_{i=1}^{N_{f}}\left[D \psi^{(i)} D \bar{\psi}^{(i)} D \chi^{(i)} D \bar{\chi}^{(i)}\right] \exp \left(-S_{\Phi}-S_{F}^{\mathrm{kin}}-S_{Y}\right)
$$

with the total action being decomposed into the Higgs action $S_{\Phi}$, the kinetic fermion action $S_{F}^{\text {kin }}$, and the Yukawa coupling term $S_{Y}$. It should be stressed once again that no gauge fields are included within this model.

The four-dimensional space-time lattice, that the model is discretized upon, is assumed to have $L$ lattice sites per dimension such that its total volume is $V=L^{4}$. Here we allow for both, finite size lattices with even $L \in \mathbb{N}$ as well as lattices with infinite extension, i.e. $L=\infty$, and we set the lattice spacing $a$ to unity for convenience. The kinetic fermion action describing the propagation of the physical fermion fields $\psi^{(i)}, \bar{\psi}^{(i)}$ is then given in the usual manner according to

$$
S_{F}^{\mathrm{kin}}=\sum_{i=1}^{N_{f}} \sum_{n, m} \bar{\psi}_{n}^{(i)} \mathcal{D}_{n, m}^{(o v)} \psi_{m}^{(i)}-2 \rho \bar{\chi}_{n}^{(i)} \mathbb{1}_{n, m} \chi_{m}^{(i)}
$$

where the four-dimensional coordinates $n, m$ as well as all field variables and coupling constants are given in dimensionless lattice units throughout this paper. Here, the (doublet) Dirac operator $\mathcal{D}^{(o v)}=\hat{\mathcal{D}}^{(o v)} \otimes \hat{\mathcal{D}}^{(o v)}$ is given by the Neuberger overlap operator $\hat{\mathcal{D}}^{(o v)}$, which is related to the Wilson operator $\hat{\mathcal{D}}^{(W)}=\gamma_{\mu}^{E} \frac{1}{2}\left(\nabla_{\mu}^{f}+\nabla_{\mu}^{b}\right)-\frac{r}{2} \nabla_{\mu}^{b} \nabla_{\mu}^{f}$ by

$$
\hat{\mathcal{D}}^{(o v)}=\rho\left\{1+\frac{\hat{A}}{\sqrt{\hat{A}^{\dagger} \hat{A}}}\right\}, \quad \hat{A}=\hat{\mathcal{D}}^{(W)}-\rho, \quad 1 \leq \rho<2 r
$$

with $\nabla_{\mu}^{f}, \nabla_{\mu}^{b}$ denoting the forward and backward difference quotients, respectively. In absence of gauge fields the eigenvectors and eigenvalues of the Neuberger operator are explicitly known. In momentum space with the allowed four-component momenta

$$
p \in \mathcal{P}=\left\{\begin{array}{cc}
(-\pi, \pi]^{\otimes 4} & : \text { for } L=\infty \\
\left\{2 \pi n / L: n \in \mathbb{N}_{0}, n<L\right\}^{\otimes 4} & : \text { for } L<\infty
\end{array}\right.
$$

the eigenvectors of the doublet operator $\mathcal{D}^{(o v)}$ are given as

$$
\Psi_{n}^{p, \zeta \epsilon k}=e^{i p \cdot n} \cdot u^{\zeta \epsilon k}(p), \quad u^{\zeta \epsilon k}(p)=\sqrt{\frac{1}{2}}\left(\begin{array}{c}
u^{\epsilon k}(p) \\
\zeta u^{\epsilon k}(p)
\end{array}\right), \quad \zeta= \pm 1, \epsilon= \pm 1, k \in\{1,2\}
$$


with $u^{\epsilon k}(p)$ denoting the usual four-component spinor structure

$$
u^{\epsilon k}(p)=\sqrt{\frac{1}{2}}\left(\begin{array}{c}
\xi_{k} \\
\epsilon \frac{\tilde{p} \bar{\Theta}}{\sqrt{\tilde{p}^{2}}} \xi_{k}
\end{array}\right) \text { for } \tilde{p} \neq 0 \quad \text { and } \quad u^{\epsilon k}(p)=\sqrt{\frac{1}{2}}\left(\begin{array}{c}
\xi_{k} \\
\epsilon \xi_{k}
\end{array}\right) \text { for } \tilde{p}=0
$$

respectively. Here $\xi_{k} \in \mathbb{C}^{2}$ are two orthonormal vectors and the four component quaternionic vectors $\Theta, \bar{\Theta}$ are defined as $\Theta=(\mathbb{1},-i \vec{\tau})$ and $\bar{\Theta}=(\mathbb{1},+i \vec{\tau})=\Theta^{\dagger}$ with $\vec{\tau}$ denoting the vector of Pauli matrices. It is well known that the eigenvalues $\nu^{ \pm}(p)$ of $\hat{\mathcal{D}}^{(o v)}$ with $\operatorname{Im}\left[\nu^{ \pm}(p)\right] \gtrless 0$ form a circle in the complex plane, the radius of which is given by the parameter $\rho$. These eigenvalues are explicitly given by

$$
\nu^{\epsilon}(p)=\rho+\rho \cdot \frac{\epsilon i \sqrt{\tilde{p}^{2}}+2 r \hat{p}^{2}-\rho}{\sqrt{\tilde{p}^{2}+\left(2 r \hat{p}^{2}-\rho\right)^{2}}}, \quad \tilde{p}_{\mu}=\sin \left(p_{\mu}\right), \quad \hat{p}_{\mu}=\sin \left(\frac{p_{\mu}}{2}\right) .
$$

The auxiliary fields $\chi^{(i)}$ on the other hand do not propagate at all and their contribution to $S_{F}^{\text {kin }}$ is chosen such that the model will obey an exact lattice chiral symmetry.

The Higgs field couples to the fermions according to the Yukawa coupling term

$$
S_{Y}=y_{N} \sum_{n, m} \sum_{i=1}^{N_{f}}\left(\bar{\psi}_{n}^{(i)}+\bar{\chi}_{n}^{(i)}\right) \underbrace{\left[\mathbb{1}_{n, m} \frac{\left(1-\gamma_{5}\right)}{2} \phi_{n}+\mathbb{1}_{n, m} \frac{\left(1+\gamma_{5}\right)}{2} \phi_{n}^{\dagger}\right]}_{B_{n, m}}\left(\psi_{m}^{(i)}+\chi_{m}^{(i)}\right)
$$

where $y_{N}$ denotes the Yukawa coupling constant and $B_{n, m}$ will be referred to as Yukawa coupling matrix. Here the Higgs field $\Phi_{n}$ is rewritten as a quaternionic, $2 \times 2$ matrix $\phi_{n}=\Phi_{n}^{0} \mathbb{1}-i \Phi_{n}^{j} \tau_{j}$ acting on the $\mathrm{SU}(2)$ index of the fermionic doublets. Due to the chiral character of this model, left- and right-handed fermions couple differently to the Higgs field, as can be seen from the appearance of the projectors $\left(1 \pm \gamma_{5}\right) / 2$ in the Yukawa term. Multiplying out the involved Gamma- and Pauli-matrices one can rewrite the Yukawa coupling matrix in the compactified form

$$
B_{m, n}=\delta_{m, n} \cdot \hat{B}\left(\Phi_{n}\right), \quad \hat{B}\left(\Phi_{n}\right)=\left(\begin{array}{cc}
\Phi_{n}^{0} \mathbb{1}+i \Phi_{n}^{3} \gamma_{5} & \Phi_{n}^{2} \gamma_{5}+i \Phi_{n}^{1} \gamma_{5} \\
-\Phi_{n}^{2} \gamma_{5}+i \Phi_{n}^{1} \gamma_{5} & \Phi_{n}^{0} \mathbb{1}-i \Phi_{n}^{3} \gamma_{5}
\end{array}\right)
$$

being block diagonal in position space. The model then obeys an exact, but lattice modified, chiral symmetry according to

$$
\begin{aligned}
& \delta \psi^{(i)}=i \epsilon\left[\gamma_{5}\left(1-\frac{1}{2 \rho} \mathcal{D}^{(o v)}\right) \psi^{(i)}+\gamma_{5} \chi^{(i)}\right], \delta \chi^{(i)}=i \epsilon \gamma_{5} \frac{1}{2 \rho} \mathcal{D}^{(o v)} \psi^{(i)}, \delta \phi=2 i \epsilon \phi \\
& \delta \bar{\psi}^{(i)}=i \epsilon\left[\bar{\psi}^{(i)}\left(1-\frac{1}{2 \rho} \mathcal{D}^{(o v)}\right) \gamma_{5}+\bar{\chi}^{(i)} \gamma_{5}\right], \delta \bar{\chi}^{(i)}=i \epsilon \bar{\psi}^{(i)} \frac{1}{2 \rho} \mathcal{D}^{(o v)} \gamma_{5}, \delta \phi^{\dagger}=-2 i \epsilon \phi^{\dagger}
\end{aligned}
$$

with $\epsilon$ denoting here the infinitesimal parameter of the chiral transformation. Since the (here omitted) lattice spacing $a$ appears in front of the Dirac operators, this exact symmetry recovers the continuum chiral symmetry (after having integrated out the auxiliary fermion fields) in the continuum limit [8]. 
Finally, we use a slightly unusual notation for the Higgs action $S_{\Phi}$ given by

$$
S_{\Phi}=-\kappa_{N} \sum_{n, \mu} \Phi_{n}^{\dagger}\left[\Phi_{n+\hat{\mu}}+\Phi_{n-\hat{\mu}}\right]+\sum_{n} \Phi_{n}^{\dagger} \Phi_{n}+\lambda_{N} \sum_{n}\left(\Phi_{n}^{\dagger} \Phi_{n}-N_{f}\right)^{2}
$$

where $\kappa_{N}$ denotes the hopping parameter and $\lambda_{N}$ is the quartic coupling constant. This notation with $N_{f}$ appearing in the quartic coupling term (which turns out to be more convenient for the later analytical considerations) can easily be shown to be equivalent to the more commonly used lattice version of the $\varphi^{4}$-action

$$
S_{\varphi}=-\kappa \sum_{n, \mu} \varphi_{n}^{\dagger}\left[\varphi_{n+\hat{\mu}}+\varphi_{n-\hat{\mu}}\right]+\sum_{n} \varphi_{n}^{\dagger} \varphi_{n}+\lambda \sum_{n}\left(\varphi_{n}^{\dagger} \varphi_{n}-1\right)^{2}
$$

by rescaling the coupling constants $\lambda, \kappa, y$ and the Higgs field $\varphi$ according to

$$
\Phi=C \cdot \varphi, \quad \lambda_{N}=\frac{\lambda}{C^{4}}, \quad \kappa_{N}=\frac{\kappa}{C^{2}}, \quad y_{N}=\frac{y}{C},
$$

where the constant $C$ has to obey the condition

$$
C^{2}-2 \lambda_{N} N_{f} C^{2}=1-2 \lambda .
$$

Furthermore, this latter action in eq. 2.13) can also easily be connected to the usual continuum notation

$$
S_{\hat{\varphi}}=\sum_{n}\left\{\frac{1}{2}\left(\nabla_{\mu}^{f} \hat{\varphi}\right)_{n}^{\dagger} \nabla_{\mu}^{f} \hat{\varphi}_{n}+\frac{1}{2} m_{0}^{2} \hat{\varphi}_{n}^{\dagger} \hat{\varphi}_{n}+\lambda_{0}\left(\hat{\varphi}_{n}^{\dagger} \hat{\varphi}_{n}\right)^{2}\right\}
$$

which explicitly involves a bare mass $m_{0}$ and the forward difference quotient $\nabla_{\mu}^{f}$. This connection is established by scaling the field and coupling constants according to

$$
\varphi_{n}=\frac{\hat{\varphi}_{n}}{\hat{C}}, \quad \lambda=\hat{C}^{4} \cdot \lambda_{0}, \quad \kappa=\frac{\hat{C}^{2}}{2}, \quad y=y_{0} \cdot \hat{C}
$$

where the constant $\hat{C}$ has to obey the relation

$$
1=\hat{C}^{2} \cdot\left(\frac{m_{0}^{2}+8}{2}+2 \lambda_{0} \hat{C}^{2}\right) .
$$

For the further analytical treatment of this model we integrate out the fermionic degrees of freedom leading to an effective Higgs model given by

$$
Z=\int D \Phi \exp \left(-S_{\text {eff }}[\Phi]\right)
$$

with the effective action $S_{\text {eff }}[\Phi]$ defined as

$$
\exp \left(-S_{\mathrm{eff}}[\Phi]\right)=\int \prod_{i=1}^{N_{f}}\left[D \psi^{(i)} D \bar{\psi}^{(i)} D \chi^{(i)} D \bar{\chi}^{(i)}\right] \exp \left(-S_{\Phi}-S_{F}^{\mathrm{kin}}-S_{Y}\right)
$$

By applying some adequate substitutions the Grassmann integrations can be performed allowing to write the effective action $S_{\text {eff }}[\Phi]$ in terms of fermionic determinants according to

$$
S_{\text {eff }}[\Phi]=S_{\Phi}[\Phi]-N_{f} \cdot \log \left[\operatorname{det}\left(y_{N} B \mathcal{D}^{(o v)}-2 \rho \mathcal{D}^{(o v)}-2 \rho y_{N} B\right)\right] .
$$




\section{Large $N_{f}$-limit for small Yukawa coupling parameters}

In this section we will derive the phase structure of the introduced Higgs-Yukawa model in the large $N_{f}$-limit for small values of the Yukawa and quartic coupling constants. The idea is to factorize the number of involved fermion doublets $N_{f}$ out of the effective action $S_{\text {eff }}[\Phi]$, since the integral over all Higgs field configurations in eq. (2.19) can then be reduced to the sum over all absolute minima of the effective action when sending $N_{f}$ to infinity. This factorization can be achieved by scaling the coupling constants and the Higgs field itself according to

$$
y_{N}=\frac{\tilde{y}_{N}}{\sqrt{N_{f}}}, \quad \lambda_{N}=\frac{\tilde{\lambda}_{N}}{N_{f}}, \quad \kappa_{N}=\tilde{\kappa}_{N}, \quad \Phi_{n}=\sqrt{N_{f}} \cdot \tilde{\Phi}_{n}
$$

where the quantities $\tilde{y}_{N}, \tilde{\lambda}_{N}, \tilde{\kappa}_{N}$, and $\tilde{\Phi}_{n}$ are kept constant in the limit $N_{f} \rightarrow \infty$.

One is thus left with the problem of finding the absolute minima of $S_{\text {eff }}[\Phi]$ in terms of the latter quantities. In general the operators $B$ and $\mathcal{D}^{(o v)}$ do not share a common eigenvector basis making the analytical evaluation of the determinant in eq. (2.21) impossible for general, space-time dependent Higgs fields. However, for sufficiently small values of the Yukawa and quartic coupling constants the kinetic term of the Higgs action becomes dominant allowing to restrict the search for the absolute minima of $S_{\text {eff }}[\Phi]$ to the ansatz

$$
\Phi_{n}=\hat{\Phi} \cdot \sqrt{N_{f}} \cdot\left(m+s \cdot(-1)^{\sum_{\mu} n_{\mu}}\right)
$$

taking only a constant and a staggered mode of the Higgs field into account. Here $\hat{\Phi} \in \mathbb{R}^{4}$ denotes a constant 4 -dimensional unit vector $(|\hat{\Phi}|=1)$, and we will refer to $m, s \in \mathbb{R}$ in the following as magnetization and staggered magnetization, respectively.

For the actual evaluation of the effective action we use the fact that the matrix $B$ now has a diagonal-plus-subdiagonal-block-structure in momentum space due to the chosen ansatz for the Higgs field according to

$$
\begin{aligned}
{\left[\mathcal{D}^{(o v)}-\frac{y_{N}}{2 \rho} B\left(\mathcal{D}^{(o v)}-2 \rho\right)\right]\left(p_{1}, p_{2}\right)=} & -\frac{\tilde{y}_{N}}{2 \rho}\left[m \cdot \delta\left(p_{1}, p_{2}\right) \cdot \hat{B}^{\left(p_{2}\right)}(\hat{\Phi}) \cdot\left(\mathcal{D}^{(o v)}\left(p_{2}\right)-2 \rho\right)\right. \\
& \left.+s \cdot \delta\left(p_{1}, \wp_{2}\right) \cdot U\left(p_{1}, p_{2}\right) \cdot \hat{B}^{\left(p_{2}\right)} \cdot\left(\mathcal{D}^{(o v)}\left(p_{2}\right)-2 \rho\right)\right] \\
& +\delta\left(p_{1}, p_{2}\right) \cdot \mathcal{D}^{(o v)}\left(p_{2}\right),
\end{aligned}
$$

where the diagonal part is caused by the constant mode of the Higgs field, while the subdiagonal contribution is created by the staggered mode. In eq. (3.3) this is expressed by $\wp_{2}$ denoting the shifted momenta $\wp_{2}=p_{2}+(\pi, \pi, \pi, \pi)$, where adequate modulo-operations are implicit to guarantee that $\wp_{2} \in \mathcal{P}$. The matrices $U\left(p_{1}, p_{2}\right), \mathcal{D}^{(o v)}(p)$, and $\hat{B}^{(p)}$ are $8 \times 8$-matrices with the indices $\zeta_{1} \epsilon_{1} k_{1}, \zeta_{2} \epsilon_{2} k_{2}$ and denote the spinor basis transformation matrix

$$
U\left(p_{1}, p_{2}\right)_{\zeta_{1} \epsilon_{1} k_{1}, \zeta_{2} \epsilon_{2} k_{2}}=\left[u^{\zeta_{1} \epsilon_{1} k_{1}}\left(p_{1}\right)\right]^{\dagger} u^{\zeta_{2} \epsilon_{2} k_{2}}\left(p_{2}\right)
$$


the Dirac matrix

$$
\mathcal{D}^{(o v)}(p)_{\zeta_{1} \epsilon_{1} k_{1}, \zeta_{2} \epsilon_{2} k_{2}}=\delta_{\epsilon_{1}, \epsilon_{2}} \cdot \delta_{k_{1}, k_{2}} \cdot \delta_{\zeta_{1}, \zeta_{2}} \cdot \nu^{\epsilon_{1}}(p)
$$

and the Yukawa coupling matrix

$$
\begin{aligned}
\hat{B}^{(p)}(\hat{\Phi})_{\zeta_{1} \epsilon_{1} k_{1}, \zeta_{2} \epsilon_{2} k_{2}} & =\left[u^{\zeta_{1} \epsilon_{1} k_{1}}(p)\right]^{\dagger} \hat{B}(\hat{\Phi}) u^{\zeta_{2} \epsilon_{2} k_{2}}(p) \\
& =\delta_{k_{1}, k_{2}}\left[\delta_{\epsilon_{1}, \epsilon_{2}} \delta_{\zeta_{1}, \zeta_{2}} \cdot \hat{\Phi}^{0}+\delta_{\epsilon_{1},-\epsilon_{2}}\left\{i \zeta_{2} \delta_{\zeta_{1}, \zeta_{2}} \hat{\Phi}^{1}+\delta_{\zeta_{1},-\zeta_{2}}\left[i \hat{\Phi}^{3}+\zeta_{2} \hat{\Phi}^{2}\right]\right\}\right],
\end{aligned}
$$

respectively. Due to this diagonal-subdiagonal-block-structure the determinant in eq. (2.21) can thus be factorized by merging the four $8 \times 8$ blocks, which correspond to the momentum indices $(p, p),(\wp, p),(p, \wp)$, and $(\wp, \wp)$. Up to some constant terms, which are independent of $\Phi$, we can thus rewrite the effective action as

$$
\begin{aligned}
S_{\mathrm{eff}}[\Phi] & =S_{\Phi}[\Phi]-N_{f} \cdot \log \left[\operatorname{det}\left(\mathcal{D}^{(o v)}-\frac{y_{N}}{2 \rho} \cdot B \cdot\left(\mathcal{D}^{(o v)}-2 \rho\right)\right)\right] \\
& =S_{\Phi}[\Phi]-N_{f} \cdot \log \left[\prod_{\substack{p \in \mathcal{P} \\
0 \leq p_{3}<\pi}} \operatorname{det}\left(\mathcal{D}^{(o v)}(p) \otimes \mathcal{D}^{(o v)}(\wp)-\frac{\tilde{y}_{N}}{2 \rho} \mathcal{M}(p)\right)\right],
\end{aligned}
$$

where the restriction $0 \leq p_{3}<\pi$ has just been introduced to prevent the double counting that would occur if one would have performed the product over all $p \in \mathcal{P}$ after having merged the blocks. Here $\mathcal{M}(p)$ denotes these merged, momentum dependent $16 \times 16$ matrices given by

$$
\mathcal{M}(p)=\left(\begin{array}{ll}
\mathcal{M}^{1,1}(p) & \mathcal{M}^{1,2}(p) \\
\mathcal{M}^{2,1}(p) & \mathcal{M}^{2,2}(p)
\end{array}\right)
$$

with

$$
\begin{aligned}
& \mathcal{M}^{1,1}(p)=m \cdot \hat{B}^{(p)}(\hat{\Phi}) \cdot\left(\mathcal{D}^{(o v)}(p)-2 \rho\right), \\
& \mathcal{M}^{1,2}(p)=s \cdot U(p, \wp) \cdot \hat{B}^{(\wp)}(\hat{\Phi}) \cdot\left(\mathcal{D}^{(o v)}(\wp)-2 \rho\right), \\
& \mathcal{M}^{2,1}(p)=s \cdot U(\wp, p) \cdot \hat{B}^{(p)}(\hat{\Phi}) \cdot\left(\mathcal{D}^{(o v)}(p)-2 \rho\right), \\
& \mathcal{M}^{2,2}(p)=m \cdot \hat{B}^{(\wp)}(\hat{\Phi}) \cdot\left(\mathcal{D}^{(o v)}(\wp)-2 \rho\right) .
\end{aligned}
$$

The expression in eq. (3.8) can be written more compactly, taking the fact into account that the matrices involved in that expression are diagonal with respect to the index $k$ due to eq. (3.5), eq. (3.6) and

$$
U(p, \wp)_{\zeta_{1} \epsilon_{1} k_{1}, \zeta_{2} \epsilon_{2} k_{2}}=\delta_{\zeta_{1}, \zeta_{2}} \cdot \delta_{\epsilon_{1},-\epsilon_{2}} \cdot \delta_{k_{1}, k_{2}} .
$$

Since one easily finds that the determinant in eq. (3.8) is invariant under the permutation $p \leftrightarrow \wp$, one can extend the product in that equation, which is performed only over one half 
of the whole momentum space, again to the full momentum space $\mathcal{P}$ by factorizing out the identity $\delta_{k_{1}, k_{2}}$. One then obtains for the effective action

$$
S_{\text {eff }}[\Phi]=S_{\Phi}[\Phi]-N_{f} \cdot \log \left[\prod_{p \in \mathcal{P}} \operatorname{det}\left(\breve{\mathcal{D}}^{(o v)}(p) \otimes \breve{\mathcal{D}}^{(o v)}(\wp)-\frac{\tilde{y}_{N}}{2 \rho} \breve{\mathcal{M}}(p)\right)\right]
$$

with the definitions

$$
\mathcal{D}^{(o v)}(p)=\delta_{k_{1}, k_{2}} \cdot \breve{\mathcal{D}}^{(o v)}(p), \quad \mathcal{M}(p)=\delta_{k_{1}, k_{2}} \cdot \breve{\mathcal{M}}(p), \text { and } \mathcal{M}^{a, b}(p)=\delta_{k_{1}, k_{2}} \cdot \breve{\mathcal{M}}^{a, b}(p),
$$

where $a, b \in\{1,2\}$. Selecting a special order for the indices $\zeta \epsilon$ according to $\{++,+-$, $-+,--\}$ the latter four $4 \times 4$ matrices are explicitly given by

$$
\begin{array}{r}
\breve{\mathcal{M}}^{1,1}(p)=m \cdot\left(\begin{array}{cccc}
\hat{\Phi}^{0} \omega^{+}(p) & i \hat{\Phi}^{1} \omega^{-}(p) & 0 & \left(i \hat{\Phi}^{3}-\hat{\Phi}^{2}\right) \omega^{-}(p) \\
i \hat{\Phi}^{1} \omega^{+}(p) & \hat{\Phi}^{0} \omega^{-}(p) & \left(i \hat{\Phi}^{3}-\hat{\Phi}^{2}\right) \omega^{+}(p) & 0 \\
0 & \left(i \hat{\Phi}^{3}+\hat{\Phi}^{2}\right) \omega^{-}(p) & \hat{\Phi}^{0} \omega^{+}(p) & -i \hat{\Phi}^{1} \omega^{-}(p) \\
\left(i \hat{\Phi}^{3}+\hat{\Phi}^{2}\right) \omega^{+}(p) & 0 & -i \hat{\Phi}^{1} \omega^{+}(p) & \hat{\Phi}^{0} \omega^{-}(p)
\end{array}\right) \\
\breve{\mathcal{M}}^{1,2}(p)=s \cdot\left(\begin{array}{cccc}
i \hat{\Phi}^{1} \omega^{+}(\wp) & \hat{\Phi}^{0} \omega^{-}(\wp) & \left(i \hat{\Phi}^{3}-\hat{\Phi}^{2}\right) \omega^{+}(\wp) & 0 \\
\hat{\Phi}^{0} \omega^{+}(\wp) & i \hat{\Phi}^{1} \omega^{-}(\wp) & 0 & \left(i \hat{\Phi}^{3}-\hat{\Phi}^{2}\right) \omega^{-}(\wp) \\
\left(i \hat{\Phi}^{3}+\hat{\Phi}^{2}\right) \omega^{+}(\wp) & 0 & -i \hat{\Phi}^{1} \omega^{+}(\wp) & \hat{\Phi}^{0} \omega^{-}(\wp) \\
0 & \left(i \hat{\Phi}^{3}+\hat{\Phi}^{2}\right) \omega^{-}(\wp) & \hat{\Phi}^{0} \omega^{+}(\wp) & -i \hat{\Phi}^{1} \omega^{-}(\wp)
\end{array}\right)
\end{array}
$$

where the abbreviation $\omega^{\epsilon}(p)=\nu^{\epsilon}(p)-2 \rho$ was used. The remaining matrices $\breve{\mathcal{M}}^{2,2}(p)$ and $\breve{\mathcal{M}}^{2,1}(p)$ are obtained from $\breve{\mathcal{M}}^{1,1}(p), \breve{\mathcal{M}}^{1,2}(p)$ by interchanging $p$ and $\wp$. Using some algebraic manipulation package, the determinant of the $8 \times 8$ matrix in eq. (3.15) can be computed leading to the final expression for the effective action

$$
\begin{aligned}
S_{\mathrm{eff}}[\Phi]= & S_{\Phi}[\Phi]-N_{f} \cdot \sum_{p \in \mathcal{P}} \log \left[\left(\left|\nu^{+}(p)\right| \cdot\left|\nu^{+}(\wp)\right|+\frac{\tilde{y}_{N}^{2}}{4 \rho^{2}}\left(m^{2}-s^{2}\right) \cdot\left|\nu^{+}(p)-2 \rho\right| \cdot\left|\nu^{+}(\wp)-2 \rho\right|\right)^{2}\right. \\
& \left.+m^{2} \frac{\tilde{y}_{N}^{2}}{4 \rho^{2}}\left(\left|\nu^{+}(p)-2 \rho\right| \cdot\left|\nu^{+}(\wp)\right|-\left|\nu^{+}(\wp)-2 \rho\right| \cdot\left|\nu^{+}(p)\right|\right)^{2}\right]^{2}
\end{aligned}
$$

With the ansatz in eq. (3.2) the Higgs field action $S_{\Phi}$ can also be written in terms of the quantities $m$ and $s$. One easily finds

$$
S_{\Phi}=N_{f} \cdot L^{4} \cdot\left\{-8 \tilde{\kappa}_{N}\left(m^{2}-s^{2}\right)+m^{2}+s^{2}+\tilde{\lambda}_{N}\left(m^{4}+s^{4}+6 m^{2} s^{2}-2\left(m^{2}+s^{2}\right)\right)\right\} .
$$

Two remarks are in order here for the orientation of the reader.

(i) The resulting phase structure in the large $N_{f}$-limit can now be obtained by minimizing the effective action with respect to $m$ and $s$. In principle one could derive the 

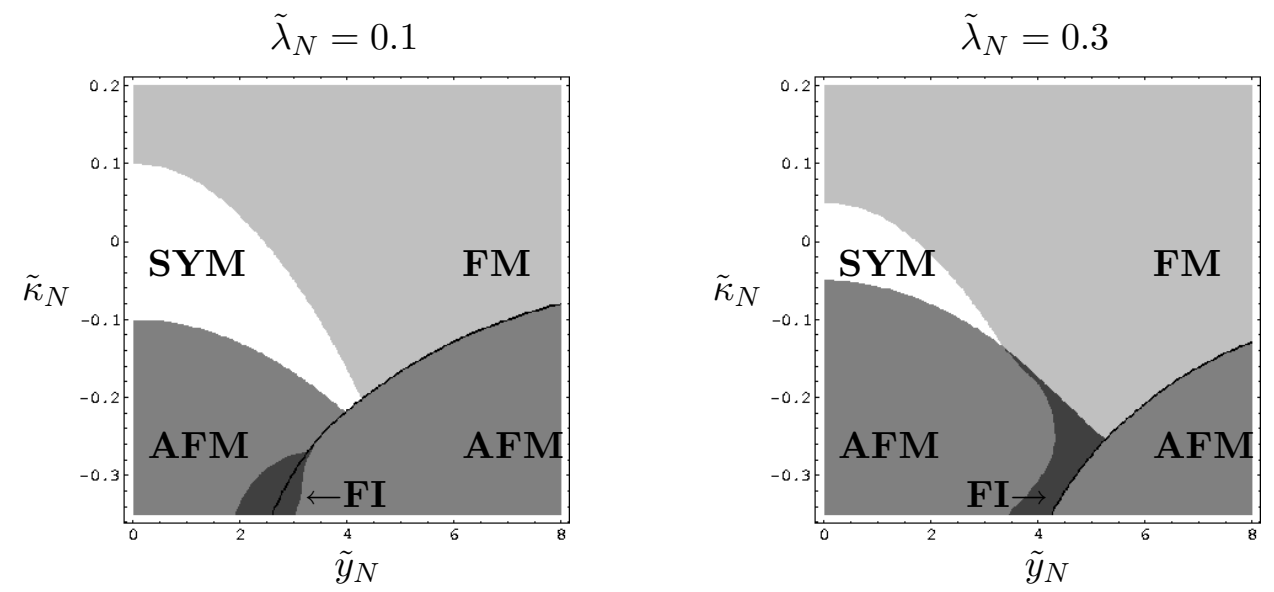

Figure 1: Phase diagrams with respect to the Yukawa coupling constant $\tilde{y}_{N}$ and the hopping parameter $\tilde{\kappa}_{N}$ for the constant quartic couplings $\tilde{\lambda}_{N}=0.1$ (left) and $\tilde{\lambda}_{N}=0.3$ (right). The black line indicates the first order phase transitions. Both phase diagrams were determined for $L=\infty$. An explanation of the occurring phases is given in the text.

corresponding phase diagrams for all values of the quartic coupling constant $\tilde{\lambda}_{N}>0$. However, as one can easily find from eq. (2.15) the case $\tilde{\lambda}_{N}>0.5$ corresponds to the strong self-coupling regime $\lambda \gg 1$ of the physically underlying $\varphi^{4}$-theory given in eq. (2.13) for large values of $N_{f}$. In that regime it is no longer reasonable to evaluate the effective action due to the strong self-interaction of the Higgs-field in that case. We therefore restrict the allowed range for the quartic coupling to $0<\tilde{\lambda}_{N}<0.5$, which corresponds to the weak self-coupling regime of the physical model in eq. (2.13).

(ii) The sum over all allowed momenta $\mathcal{P}$ in eq. (3.19) becomes a four-dimensional momentum integral over $\mathcal{P}$ for $L=\infty$ according to

$$
\frac{1}{L^{4}} \sum_{p \in \mathcal{P}} \ldots \rightarrow \int_{p \in \mathcal{P}} \frac{d^{4} p}{(2 \pi)^{4}} \cdots
$$

which was actually used in the numerical evaluation of the effective action.

We now present the phase diagrams for $\tilde{\lambda}_{N}=0.1$ and $\tilde{\lambda}_{N}=0.3$ in figure 1. These phase diagrams were calculated for an infinite lattice, i.e. for $L=\infty$. Here we distinguish between the following four phases:

(i) The symmetric phase (SYM): $m=0, s=0$

(ii) The ferromagnetic phase (FM): $m \neq 0, s=0$

(iii) The anti-ferromagnetic phase (AFM): $m=0, s \neq 0$

(iv) The ferrimagnetic phase (FI): $m \neq 0, s \neq 0$ 
In both cases, i.e. $\tilde{\lambda}_{N}=0.1$ and $\tilde{\lambda}_{N}=0.3$, one finds a symmetric phase approximately centered around $\tilde{\kappa}_{N}=0$ at sufficiently small values of the Yukawa coupling constant $\tilde{y}_{N}$, as one would have expected, since the model becomes the pure $\phi^{4}$-theory in the limit $\tilde{y}_{N} \rightarrow 0$. From the same consideration one would also expect the accompanying phase transitions to be of second order. This is indeed the case as can clearly be seen in figure 2 showing the expectation values of the amplitudes $m$ and $s$ for different values of $\tilde{y}_{N}$ as obtained in the minimization process. With increasing $\tilde{y}_{N}$ the symmetric phase bends downwards to negative values of the hopping parameter $\tilde{\kappa}_{N}$, unless it either hits a first order phase transition to an anti-ferromagnetic phase (black line in figure 1), the order of which can be determined from figure 3 (this is the case for $\tilde{\lambda}_{N}=0.1$ ), or it eventually goes over into two FM-FI and FI-AFM second order phase transitions, which is the case for $\tilde{\lambda}_{N}=0.3$.

Here we present only the expectation values of $m$ and $s$ for $\tilde{\lambda}_{N}=0.3$ and not for $\tilde{\lambda}_{N}=$ 0.1 , since the latter plots would not provide qualitatively new information to the reader.

Interestingly, the ferrimagnetic phase (FI) exists in both presented scenarios, i.e. for $\tilde{\lambda}_{N}=0.1$ and $\tilde{\lambda}_{N}=0.3$, even deeply inside the anti-ferromagnetic phase region in the neighbourhood of the first order phase transition boundary.

\section{Large $N_{f}$-limit for large Yukawa coupling parameters}

In this section we will examine the phase diagram of the considered Higgs-Yukawa model in the regime of large values of the Yukawa coupling constant $y_{N}$ and for arbitrary values of the quartic coupling constant $\lambda_{N}>0$. This will be done in three steps. Firstly, the effective action is expanded in powers of the inverse coupling constant $1 / y_{N}$. Taking only the first non-vanishing contribution of this power series into account and performing the large $N_{f}$-limit in such a way, that the amplitude of the Higgs field is fixed, the model then effectively becomes an $O(4)$-symmetric, non-linear sigma-model up to some finite volume terms. Finally, the phase diagram of the latter sigma-model is determined by an additional large $N$-limit, where $N$ denotes here the number of Higgs field components.

For an evaluation of the effective action it is crucial to pay special attention to the fermion doubler modes

$$
\mathcal{Q}_{\pi}=\left\{\Psi^{p, \zeta \epsilon k}: p_{\mu} \in\{0, \pi\}, p \neq 0, \zeta, \epsilon= \pm 1, k \in\{1,2\}\right\}
$$

which we will refer to as $\pi$-modes in the following. Given these 120 modes one can define the corresponding projection operator

$$
P_{\pi}=\sum_{\Psi \in \mathcal{Q}_{\pi}} \Psi \Psi^{\dagger}
$$

projecting to the sub-space $V_{\pi}=\operatorname{span}\left(\mathcal{Q}_{\pi}\right)$ spanned by $\mathcal{Q}_{\pi}$. Using this notation one can easily establish the very helpful relation

$$
\begin{aligned}
\operatorname{det}\left(E\left(\mathbb{1}-P_{\pi}\right)+P_{\pi} F P_{\pi}\right) & =\operatorname{det}\left(\left(\mathbb{1}-P_{\pi}\right) E\left(\mathbb{1}-P_{\pi}\right)+P_{\pi} F P_{\pi}\right) \\
& =\operatorname{det}\left(\left(\mathbb{1}-P_{\pi}\right) E+P_{\pi} F P_{\pi}\right)=\operatorname{det}^{\prime}(E) \cdot \operatorname{det}^{*}(F)
\end{aligned}
$$




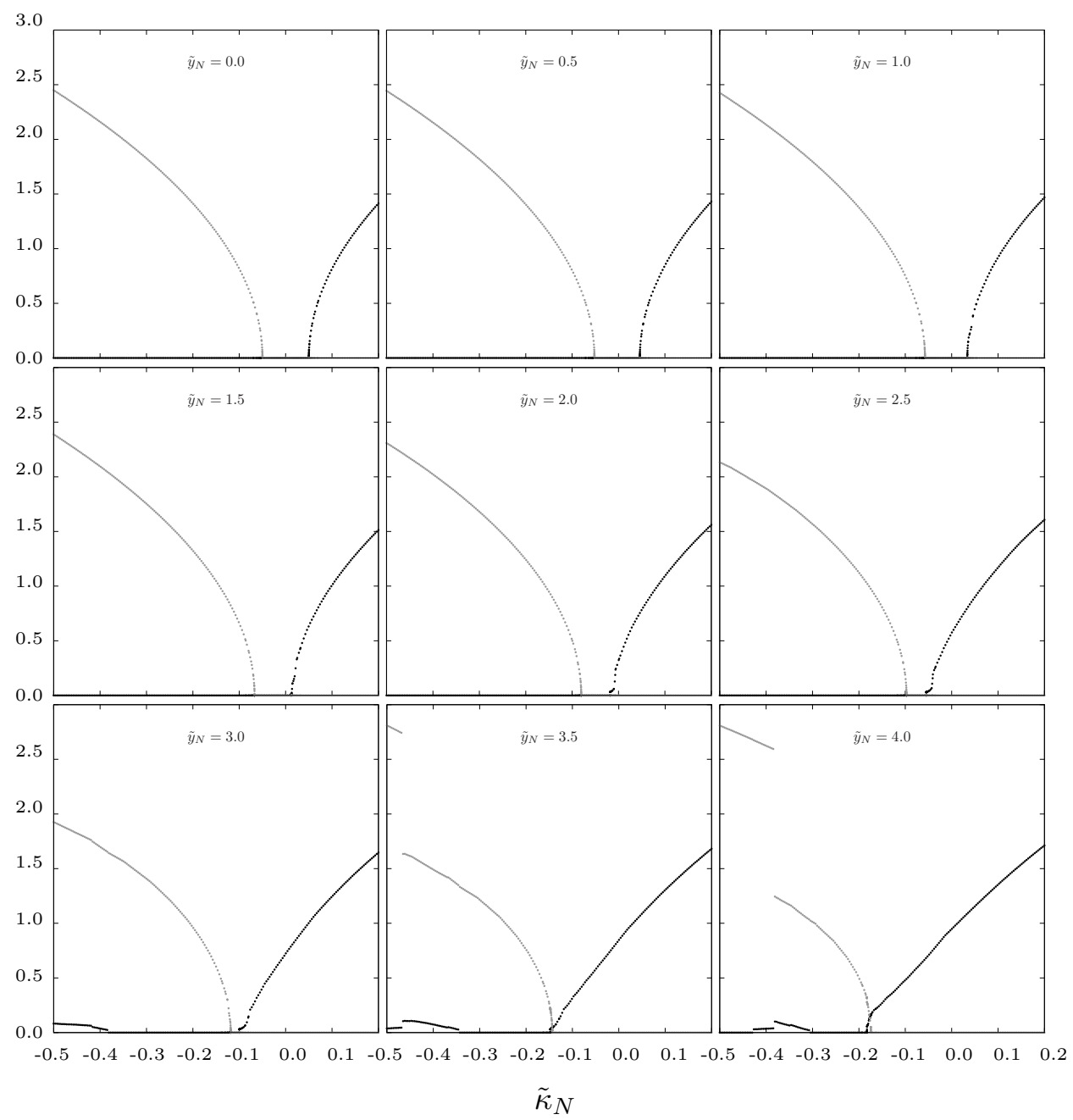

Figure 2: Expectation values for the amplitudes of the constant ( $m$ : black curve) and staggered ( $s$ : gray curve) modes for several selected values of the Yukawa coupling constant $\tilde{y}_{N}$ and a constant quartic coupling $\tilde{\lambda}_{N}=0.3$. The results were obtained for $L=\infty$.

where $E$ and $F$ are arbitrary operators defined on the same space $V$ as $\mathcal{D}^{(o v)}$ and $B$. Here the expression $\operatorname{det}^{*}(F)$ denotes the determinant of $F$ with respect to the sub-space $V_{\pi}$ and $\operatorname{det}^{\prime}(E)$ is the determinant of $E$ with respect to the complementary space $V / V_{\pi} \equiv$ $\operatorname{span}\left(\mathcal{Q} / \mathcal{Q}_{\pi}\right)$, where $\mathcal{Q}$ denotes the full set of all modes. Using eq. (4.3) several times one can rewrite the effective action according to

$$
\begin{aligned}
e^{-\frac{S_{\mathrm{eff}}[\Phi]-S_{\Phi}}{N_{f}}} & =\operatorname{det}\left(y_{N} B\left(\mathcal{D}^{(o v)}-2 \rho\right)-2 \rho \mathcal{D}^{(o v)}\right) \\
& =\left(-4 \rho^{2}\right)^{120} \cdot \operatorname{det}^{\prime}\left(y_{N} B^{\prime}\left(\mathcal{D}^{\prime(o v)}-2 \rho \mathbb{1}^{\prime}\right)-2 \rho \mathcal{D}^{\prime(o v)}\right) \\
& =\left(-4 \rho^{2}\right)^{120} \cdot \operatorname{det}^{\prime}\left(y_{N}\right) \cdot \operatorname{det}^{\prime}\left(\mathcal{D}^{\prime(o v)}-2 \rho \mathbb{1}^{\prime}\right) \cdot \operatorname{det}^{\prime}\left(B^{\prime}-\frac{2 \rho}{y_{N}} \mathcal{D}^{\prime(o v)}\left(\mathcal{D}^{\prime(o v)}-2 \rho \mathbb{1}^{\prime}\right)^{-1}\right) \\
& =\text { Const } \cdot \operatorname{det}\left(B-(B-\mathbb{1}) P_{\pi}-\frac{2 \rho}{y_{N}} \mathcal{A}\right) \\
& =\text { Const } \cdot \operatorname{det}(B) \cdot \operatorname{det}\left(\mathbb{1}-\left(\mathbb{1}-B^{-1}\right) P_{\pi}\right) \cdot \operatorname{det}\left(\mathbb{1}-\frac{2 \rho}{y_{N}} B^{-1} \mathcal{A}\left[\mathbb{1}-\left(\mathbb{1}-B^{-1}\right) P_{\pi}\right]^{-1}\right)
\end{aligned}
$$




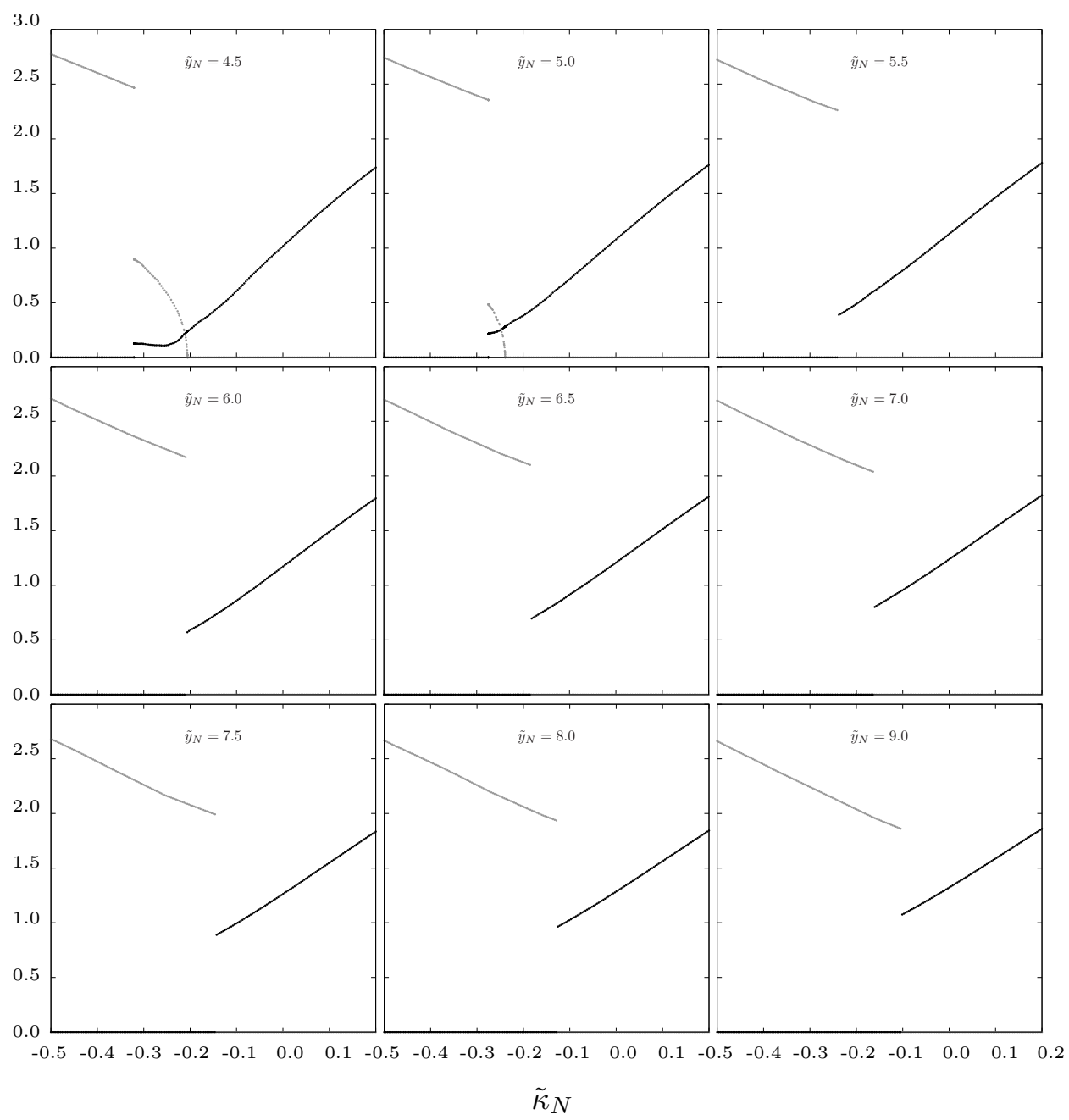

Figure 3: Expectation values for the amplitudes of the constant ( $m$ : black curve) and staggered ( $s$ : gray curve) modes for several selected values of the Yukawa coupling constant $\tilde{y}_{N}$ and a constant quartic coupling $\tilde{\lambda}_{N}=0.3$. The results were obtained for $L=\infty$.

where $\mathcal{D}^{\prime(o v)}, B^{\prime}$, and $\mathbb{1}^{\prime}$ denote the restriction of the operators $\mathcal{D}^{(o v)}, B$, and $\mathbb{1}$ to the subspace $V / V_{\pi}$. This restriction is introduced, since it guarantees $\mathcal{D}^{\prime(o v)}-2 \rho \mathbb{1}^{\prime}$ to be invertible. The operator $\mathcal{A}$ is then defined by extending the domain of the inverse of $\mathcal{D}^{\prime(o v)}-2 \rho \mathbb{1}^{\prime}$ again to the full space $V$ by inserting the projector $\mathbb{1}-P_{\pi}$ according to

$$
\mathcal{A}=\mathcal{D}^{\prime(o v)} \cdot\left[\mathcal{D}^{\prime(o v)}-2 \rho \mathbb{1}^{\prime}\right]^{-1} \cdot\left(\mathbb{1}-P_{\pi}\right),
$$

which is well-defined and finite over the whole space $V$. The last determinant in eq. (4.4) can further be reduced by using the result

$$
\begin{aligned}
{\left[\mathbb{1}-\left(\mathbb{1}-B^{-1}\right) P_{\pi}\right]^{-1}=} & \mathbb{1}-P_{\pi}+P_{\pi}\left(1-P_{\pi}+P_{\pi} B^{-1} P_{\pi}\right)^{-1} P_{\pi} \\
& -\left(\mathbb{1}-P_{\pi}\right) B^{-1} P_{\pi}\left(1-P_{\pi}+P_{\pi} B^{-1} P_{\pi}\right)^{-1} P_{\pi}
\end{aligned}
$$


and by applying again relation (4.3) leading to the compact notation for the effective action

$$
\begin{aligned}
S_{\mathrm{eff}}[\Phi]=S_{\Phi} & -N_{f} \cdot \log \operatorname{det}(B)-N_{f} \cdot \log \operatorname{det}^{*}\left(B^{-1}\right)-N_{f} \cdot \log \operatorname{det}^{*}\left(\mathbb{1}+\frac{2 \rho}{y_{N}} F[\Phi]\right) \\
& -N_{f} \cdot \log \operatorname{det}\left(\mathbb{1}-\frac{2 \rho}{y_{N}} \mathcal{A} \cdot B^{-1}\right)
\end{aligned}
$$

with the abbreviation $F[\Phi]$ defined as the somewhat lengthy expression

$$
F[\Phi]=\left[\mathbb{1}-\frac{2 \rho}{y_{N}} B^{-1} \mathcal{A}\right]^{-1} B^{-1} \mathcal{A} B^{-1} P_{\pi}\left[\mathbb{1}-P_{\pi}+P_{\pi} B^{-1} P_{\pi}\right]^{-1} .
$$

However, the latter determinants det* only give rise to some finite volume effects, since these determinants are only performed over the 120-dimensional sub-space $V_{\pi}$. Their contributions to the effective action do therefore not scale proportional to $L^{4}$ as the lattice size increases in contrast to all other appearing terms. We will come back to discussing these finite volume effects later. Here, we will first continue with the evaluation of the last term in eq. (4.7) by rewriting the corresponding trace as a power series in the inverse coupling constant $1 / y_{N}$ according to

$$
\operatorname{Tr} \log \left(\mathbb{1}-\frac{2 \rho}{y_{N}} \mathcal{A} \cdot B^{-1}\right)=-\operatorname{Tr} \sum_{r=1}^{\infty} \frac{2^{r}}{r}\left(\frac{\rho}{y_{N}}\right)^{r}\left[\mathcal{A} B^{-1}\right]^{r}
$$

and by eventually cutting off this power series after the first non-vanishing term, which is well-justified for sufficiently large $y_{N}$. For our purpose of establishing the desired connection to a sigma-model it is most convenient to evaluate these expressions in position space. Here the matrix $B^{-1}$ is block diagonal and explicitly given by

$$
B^{-1}=B^{\dagger} \cdot\left(B B^{\dagger}\right)^{-1}, \quad B_{m, n}^{-1}=\delta_{m, n} \cdot \hat{B}\left(\Phi_{n}^{*} /\left|\Phi_{n}\right|^{2}\right),
$$

where the notation $\left(\Phi_{n}^{*}\right)^{0}=\Phi_{n}^{0},\left(\Phi_{n}^{*}\right)^{i}=-\Phi_{n}^{i}$ was used and $\hat{B}$ was defined in eq. (2.9). In position space the matrix $\mathcal{A} B^{-1}$ can hence be written as

$$
\begin{aligned}
{\left[\mathcal{A} B^{-1}\right]_{n_{1}, n_{2}} } & =\sum_{p \in \mathcal{P}} \sum_{\zeta \epsilon k} \frac{e^{i p n_{1}} u^{\zeta \epsilon k}(p) \alpha^{\epsilon}(p) e^{-i p n_{2}}\left[u^{\zeta \epsilon k}(p)\right]^{\dagger}}{\left|\Psi^{p, \zeta \epsilon k}\right|^{2}} \hat{B}\left(\Phi_{n_{2}}^{*} /\left|\Phi_{n_{2}}\right|^{2}\right) \\
& =\frac{1}{L^{4}} \sum_{p \in \mathcal{P}} \sum_{\substack{\zeta \epsilon k \\
\zeta^{\prime} \epsilon^{\prime} k^{\prime}}} \alpha^{\epsilon}(p) e^{i p\left(n_{1}-n_{2}\right)} u^{\zeta \epsilon k}(p)\left(\hat{B}^{(p)}\left(\Phi_{n_{2}}^{*} /\left|\Phi_{n_{2}}\right|^{2}\right)\right)_{\zeta \epsilon k, \zeta^{\prime} \epsilon^{\prime} k^{\prime}}\left[u^{\zeta^{\prime} \epsilon^{\prime} k^{\prime}}(p)\right]^{\dagger}
\end{aligned}
$$

with $\hat{B}^{(p)}$ as defined in eq. (3.6). The scalars $\alpha^{\epsilon}(p)$ denote the eigenvalues of the antihermitian operator $\mathcal{A}$ corresponding to its eigenvectors $\Psi^{p, \zeta \epsilon k}$ and are explicitly given by

$$
i \mathbb{R} \ni \alpha^{\epsilon}(p)=\left\{\begin{array}{cl}
\frac{\nu^{\epsilon}(p)}{\nu^{\epsilon}(p)-2 \rho} & : p \in \mathcal{P}, \nu^{\epsilon}(p) \neq 2 \rho \\
0 & : p \in \mathcal{P}, \nu^{\epsilon}(p)=2 \rho
\end{array} .\right.
$$

The result for the trace of the operator $\mathcal{A} B^{-1}$ is then directly found to be

$$
\operatorname{Tr}\left[\mathcal{A} B^{-1}\right]=\frac{1}{L^{4}} \sum_{n} \sum_{p \in \mathcal{P}} \operatorname{Tr}_{8 \times 8}\left[\left|\Phi_{n}\right|^{-2} \mathcal{A}(p) \hat{B}^{(p)}\left(\Phi_{n}^{*}\right)\right],
$$


which can be generalized to the trace of the $r$-th power of $\mathcal{A} B^{-1}$ yielding

$$
\operatorname{Tr}\left[\mathcal{A} B^{-1}\right]^{r}=\sum_{\substack{n_{1}, \ldots, n_{r} \\ p_{1}, \ldots, p_{r} \in \mathcal{P}}} \operatorname{Tr}_{8 \times 8}\left[\prod_{i=1}^{r} \frac{e^{i p_{i}\left(n_{i}-n_{i+1}\right)}}{L^{4}}\left|\Phi_{n_{i+1}}\right|^{-2} \mathcal{A}\left(p_{i}\right)\left(\hat{B}^{\left(p_{i}\right)}\left(\Phi_{n_{i+1}}^{*}\right)\right) U\left(p_{i}, p_{i+1}\right)\right]
$$

where $p_{n+1}$ is identified with $p_{1}$, and $x_{n+1}$ with $x_{1}$, and the expression $\mathcal{A}(p)$ stands for the diagonal matrix

$$
\mathcal{A}(p)_{\zeta_{1} \epsilon_{1} k_{1}, \zeta_{2} \epsilon_{2} k_{2}}=\delta_{\zeta_{1}, \zeta_{2}} \cdot \delta_{\epsilon_{1}, \epsilon_{2}} \cdot \delta_{k_{1}, k_{2}} \cdot \alpha^{\epsilon_{1}}(p) .
$$

At this point we refer the interested reader to appendix $\mathrm{A}$ for the details of this calculation in order to sustain the readability of this text.

However, it turns out that the evaluation of eq. (4.14) becomes much easier, if one inserts the identity $U\left(p_{i}, 0\right) U\left(0, p_{i}\right)$ at some proper places. The remaining $8 \times 8$ trace can then be simplified to

$$
\operatorname{Tr}_{8 \times 8}\left[\prod_{i=1}^{r} \mathcal{A}\left(p_{i}\right)\left(\hat{B}^{\left(p_{i}\right)}\left(\Phi_{n_{i+1}}^{*}\right)\right) U\left(p_{i}, p_{i+1}\right)\right]=\operatorname{Tr}_{8 \times 8}\left[\prod_{i=1}^{r} \mathcal{A}^{(0)}\left(p_{i}\right)\left(\hat{B}^{(0)}\left(\Phi_{n_{i+1}}^{*}\right)\right)\right]
$$

where the representation $\hat{B}^{(0)}\left(\Phi_{n}^{*}\right)$ of the Yukawa coupling matrix can directly be taken from eq. (3.6) and $\mathcal{A}^{(0)}(p)$ is given by

$$
\begin{aligned}
\mathcal{A}^{(0)}(p) & =U(0, p) \mathcal{A}(p) U(p, 0) \\
& =\frac{\alpha^{+}(p)}{\sqrt{\tilde{p}^{2}}} \cdot\left(\begin{array}{cc}
\tilde{p}_{0} & -\overrightarrow{\tilde{p}} \vec{\Theta} \\
\overrightarrow{\tilde{p}} \vec{\Theta} & -\tilde{p}_{0}
\end{array}\right) \otimes\left(\begin{array}{cc}
\tilde{p}_{0} & -\overrightarrow{\tilde{p}} \vec{\Theta} \\
\overrightarrow{\tilde{p}} \vec{\Theta} & -\tilde{p}_{0}
\end{array}\right)
\end{aligned}
$$

where the relation $\alpha^{+}(p)=-\alpha^{-}(p)$ has implicitly been used. Due to the insertion of the spinor basis transformation matrices $U\left(p_{i}, 0\right)$ and $U\left(0, p_{i}\right)$ the sums over the momenta in eq. (4.14) factorize now according to

$$
\operatorname{Tr}\left[\mathcal{A} B^{-1}\right]^{r}=\sum_{n_{1}, \ldots, n_{r}} \operatorname{Tr} r_{8 \times 8}[\prod_{i=1}^{r} \underbrace{\left(\sum_{p_{i} \in \mathcal{P}} \frac{e^{i p_{i}\left(n_{i}-n_{i+1}\right)}}{L^{4}} \mathcal{A}^{(0)}\left(p_{i}\right)\right)\left|\Phi_{n_{i+1}}\right|^{-2}\left(\hat{B}^{(0)}\left(\Phi_{n_{i+1}}^{*}\right)\right)}_{\mathcal{T}_{n_{i}, n_{i+1}}}]
$$

where each momentum sum is a four-dimensional Fourier transform of an anti-symmetric and purely imaginary summand, hence yielding real values. With the definition

$$
\mathbb{R} \ni \Gamma_{\mu}(\Delta n)=-\Gamma_{\mu}(-\Delta n)=\sum_{p \in \mathcal{P}} \frac{e^{i p \Delta n}}{L^{4}} \alpha^{+}(p) \cdot \frac{\tilde{p}_{\mu}}{\sqrt{\tilde{p}^{2}}}
$$


the hermitian matrix $\mathcal{T}_{n, m}$ appearing in eq. (4.18) can compactly be written as

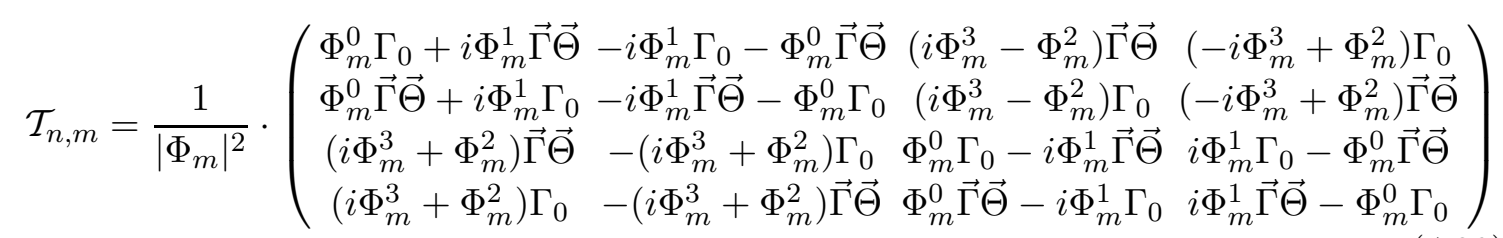

with the abbreviations $\Gamma_{\mu} \equiv \Gamma_{\mu}(\Delta n)$ and $\Delta n=n-m$. Therefore, the first order summand of the power series in eq. (4.9) reading

$$
\operatorname{Tr}\left[\mathcal{A B} B^{-1}\right]=\sum_{n} \operatorname{Tr}_{8 \times 8}\left[\mathcal{T}_{n, n}\right]=0
$$

is identical to zero and the first non-vanishing contribution is the second order term, which can be evaluated by explicitly computing the $8 \times 8$ trace, yielding

$$
\begin{aligned}
\operatorname{Tr}\left[\mathcal{A} B^{-1}\right]^{2} & =\sum_{n_{1}, n_{2}} \operatorname{Tr}_{8 \times 8}\left[\mathcal{T}_{n_{1}, n_{2}} \mathcal{T}_{n_{2}, n_{1}}\right] \\
& =-8 \cdot \sum_{n_{1}, n_{2}} \frac{\Phi_{n_{1}}^{\mu} \Phi_{n_{2}}^{\mu}}{\left|\Phi_{n_{1}}\right|^{2} \cdot\left|\Phi_{n_{2}}\right|^{2}} \cdot|\Gamma(\Delta n)|^{2} .
\end{aligned}
$$

Cutting off the power series in eq. (4.9) after this first non-vanishing term, which is well justified for sufficiently large values of the Yukawa coupling constant, the effective action can be written as

$$
\begin{aligned}
S_{\text {eff }}[\Phi]= & S_{\Phi}-N_{f} \cdot\left(\sum_{n} \log \left(\left|\Phi_{n}\right|^{8}\right)+\frac{(4 \rho)^{2}}{y_{N}^{2}} \sum_{n_{1}, n_{2}}|\Gamma(\Delta n)|^{2} \frac{\Phi_{n_{1}}^{\dagger} \Phi_{n_{2}}}{\left|\Phi_{n_{1}}\right|^{2} \cdot\left|\Phi_{n_{2}}\right|^{2}}\right) \\
& -N_{f} \cdot \log \operatorname{det}^{*}\left(B^{-1}\right)-N_{f} \cdot \log \operatorname{det}^{*}\left(\mathbb{1}+\frac{2 \rho}{y_{N}} F[\Phi]\right)
\end{aligned}
$$

where the matrix $F[\Phi]$ has been defined in eq. (4.8).

Some remarks concerning the remaining determinants in the latter result are in order here for the orientation of the reader. Here det* denotes the determinant over the sub-space $V_{\pi}$, which has the dimension 120 . In contrast to all other terms appearing in the effective action these determinants are not proportional to $L^{4}$. They are therefore suppressed as the lattice size $L$ goes to infinity. Moreover, the very last term in eq. 4.23) even vanishes on finite lattices when the Yukawa coupling constant $y_{N}$ becomes large. This is in contrast to the determinant $\operatorname{det}^{*}\left(B^{-1}\right)$ being independent of $y_{N}$. However, it is nevertheless quite instructive to consider these finite volume effects in more detail. This can at least be done for the first determinant $\operatorname{det}^{*}\left(B^{-1}\right)$, which can be exactly evaluated for the ansatz given in eq. (3.2) taking only a constant and a staggered mode of the Higgs field into account. In that case the inverse of $B$ can also be described in terms of a constant and a staggered mode according to

$$
\Phi_{n} /\left|\Phi_{n}\right|^{2}=\hat{\Phi} \cdot N_{f}^{-\frac{1}{2}} \cdot\left(\tilde{m}+\tilde{s} \cdot(-1)^{\sum_{\mu} n_{\mu}}\right), \quad \tilde{m}=\frac{m}{m^{2}-s^{2}}, \quad \tilde{s}=\frac{s}{s^{2}-m^{2}}
$$


which allows to determine the desired determinant in a similar manner as described in section 3 yielding

$$
\log \operatorname{det}^{*}\left(B^{-1}\right)=-60 \log \left(N_{f}\right)+8 \log |\tilde{m}|+56 \log \left|\tilde{m}^{2}-\tilde{s}^{2}\right| .
$$

The obvious asymmetry in $m$ and $s$ is caused by the fact that the 8 zero modes $\Psi^{0, \zeta \epsilon k}$ are not included in the sub-space $V_{\pi}$. The effect of the latter terms and especially the asymmetry in $m$ and $s$ is clearly observed in corresponding Monte-Carlo simulations 16. on small lattices and large values of the Yukawa coupling constant $y_{N}$. Moreover, the result in eq. (4.25) would also hinder the expectation value of the Higgs field from vanishing, thus obscuring the potential existence of symmetric phases at large $y_{N}$ on small lattices. However, as the lattice size increases these finite volume effects eventually disappear. In the following we will therefore neglect the det* terms in the effective action (4.23), which is well justified on sufficiently large lattices.

To establish the announced connection to a sigma-model we now consider the large $N_{f}$-limit where the coupling constants scale according to

$$
y_{N}=\tilde{y}_{N}, \tilde{y}_{N}=\mathrm{const}, \quad \lambda_{N}=\frac{\tilde{\lambda}_{N}}{N_{f}}, \tilde{\lambda}_{N}=\mathrm{const}, \quad \kappa_{N}=\frac{\tilde{\kappa}_{N}}{N_{f}}, \tilde{\kappa}_{N}=\mathrm{const},
$$

and for the Higgs field we consider an ansatz in which the amplitude of the local vectors $\Phi_{n}$ is fixed to $\varphi \in \mathbb{R}$ according to

$$
\Phi_{n}=\sqrt{N_{f}} \cdot \varphi \cdot \sigma_{n}, \quad\left|\sigma_{n}\right|=1
$$

where the four-component, space-time position dependent unit vectors $\sigma_{n}$ are arbitrary. In this setting the contributions to the (reduced) effective action are either of order $O\left(N_{f}\right)$ or $O(1)$. Considering only the leading order terms, for which the fermion doublet number $N_{f}$ can be completely factorized out, then allows to fix the Higgs field amplitude $\varphi$ by the determination equation

$$
0=-4 \cdot \frac{1}{\varphi^{2}}+1+2 \tilde{\lambda}_{N} \cdot\left(\varphi^{2}-1\right)
$$

With this fixation of the Higgs field amplitude the model in eq. (4.23) becomes effectively a non-local, four-dimensional, non-linear sigma-model in the large $N_{f}$-limit given by

$$
S_{\mathrm{eff}}=-\sum_{n_{1}, n_{2}} \kappa_{n_{1}, n_{2}}^{\mathrm{eff}} \cdot \sigma_{n_{1}}^{\dagger} \sigma_{n_{2}}
$$

with the effective, non-local coupling matrix

$$
\kappa_{n_{1}, n_{2}}^{\mathrm{eff}}=\frac{16 \rho^{2}}{\tilde{y}_{N}^{2} \varphi^{2}}|\Gamma(\Delta n)|^{2}+\tilde{\kappa}_{N} \cdot \varphi^{2} \cdot \sum_{\mu= \pm 1}^{ \pm 4} \delta_{\Delta n, \hat{e}_{\mu}} .
$$

Here the notation "non-local" simply refers to the fact, that the field $\sigma_{n}$ at any lattice site $n$ couples itself to any other site of the lattice. This leaves nevertheless open the possibility that the interaction is local in a field theoretical sense with exponentially decaying coupling 
strength [17]. We did, however, not investigate the question in this paper, since eventually we are mostly interested in the small Yukawa coupling region.

Basically, the outcome in eq. (4.30) reproduces the result which was found for a HiggsYukawa model based on Wilson fermions [12] with the only difference that the coupling matrix in that case consisted only of nearest-neighbour couplings.

The phase diagram of the obtained sigma-model (4.29) can be determined analytically by an additional large $N$-limit with $N$ denoting here the number of components of the vectors $\sigma_{n}$. The first step towards this evaluation is to remove the restriction $\left|\sigma_{n}\right|=1$ by introducing an auxiliary one-component, real field $\lambda_{n}$. This can be done at least in two ways. One can either encode the restriction $\left|\sigma_{n}\right|=1$ as a $\delta$-function [18] written in terms of an integration of $\exp \left(i \lambda_{n}\left(\left|\sigma_{n}\right|^{2}-1\right)\right)$ over $\lambda_{n}$, or alternatively, one can address this restriction by introducing the field variables $\lambda_{n}$ as Lagrange-multipliers [19]. Here we follow the latter approach which leads us to the extended action

$$
S[\sigma, \lambda]=\frac{1}{t_{N}} \cdot\left\{\sum_{n_{1}, n_{2}} \sum_{i=1}^{N}-\kappa_{n_{1}, n_{2}}^{\mathrm{eff}} \cdot \sigma_{n_{1}}^{i} \cdot \sigma_{n_{2}}^{i}+\sum_{n} \lambda_{n} \cdot\left(\sum_{i=1}^{N}\left[\sigma_{n}^{i}\right]^{2}-1\right)\right\}
$$

the minima of which can now be searched for without having to consider any restriction on the Higgs field amplitude. Here, an additional parameter $t_{N}$ was introduced. For $t_{N}=1$ the given action corresponds to the prior form of the action. This new parameter is inserted, since it will allow to factorize a factor $N$ out of the action as required by the large $N$ approach. This can be achieved by scaling $t_{N}$ according to

$$
t_{N}=\frac{\tilde{t}_{N}}{N}, \quad \tilde{t}_{N}=\mathrm{const}
$$

where we choose the setting $\tilde{t}_{N}=4$, since this recovers our actual effective sigma-model at $N=4$.

The remaining problem to solve is to find the minimum of the action $S[\sigma, \lambda]$. However, it is well known from investigations of pure sigma-models that the phase transitions of such models cannot be correctly determined by evaluating the effective action $S[\sigma, \lambda]$ in eq. (4.31) directly by restricting the consideration to only some selected modes of the fields $\sigma$ and $\lambda$. (Doing so would yield a first order phase transition at $\tilde{\kappa}_{N}=0$.) This is in contrast to the situation we discussed in section 3. Instead, we first integrate out all modes of all $N$ components of the field $\sigma$ except for the constant and staggered modes. This can be done by taking only the constant mode of the auxiliary field $\lambda_{n}$ into account, i.e. $\lambda_{n} \equiv \lambda=$ const. Doing so reduces the action $S[\sigma, \lambda]$ to

$$
\begin{aligned}
S\left[m^{i}, s^{i}, \lambda\right]=-\ln & {\left[\operatorname{det}^{\prime}\left(-\kappa^{\mathrm{eff}}+\lambda\right)\right]^{-N / 2}+\frac{1}{t_{N}}\left\{\sum_{i=1}^{N}\left[m^{i}\right]^{2} \cdot\left\langle 0\left|-\kappa^{\mathrm{eff}}+\lambda\right| 0\right\rangle\right.} \\
& \left.+\sum_{i=1}^{N}\left[s^{i}\right]^{2} \cdot\left\langle\pi\left|-\kappa^{\mathrm{eff}}+\lambda\right| \pi\right\rangle-L^{4} \lambda\right\},
\end{aligned}
$$

depending only on the real scalar $\lambda$ and the amplitudes $m^{i}, s^{i}$ of the constant and staggered modes, respectively. Here the notations $|0\rangle$ and $|\pi\rangle$ were used, denoting the constant and 
staggered modes (normalized by a factor $1 / \sqrt{L^{4}}$ ) according to

$$
|k\rangle \equiv \sqrt{\frac{1}{L^{4}}} e^{i k \cdot n}
$$

being eigenvectors of $\kappa^{\text {eff }}$ and det $^{\prime}$ is the determinant neglecting the two latter modes. For convenience, the introduced short-hand notation $0 \equiv(0,0,0,0)$ and $\pi \equiv(\pi, \pi, \pi, \pi)$ will also be applied in the following where it is unambiguous.

One remark is in order here for the orientation of the reader. The performed Gaussintegrations are only well-defined, if the involved eigenvalues of the operator $-\kappa^{\text {eff }}+\lambda$ are positive, which is not guaranteed at this point. However, this step will be justified (and made more precise) a posteriori when a certain value for the scalar $\lambda$ will be assumed by solving the resulting gap equations. Here we will first continue with this formal expression and postpone its further discussion to the end of this section.

To evaluate this latter determinant, the eigenvalues of the matrix $\kappa^{\text {eff }}$ need to be known. The eigenvectors are simply plane waves with wave vectors $k \in \mathcal{P}$ and one easily finds the corresponding eigenvalues according to

$$
\sum_{n_{2}} \kappa_{n_{1}, n_{2}}^{\mathrm{eff}} \cdot e^{i k n_{2}}=\left(2 \tilde{\kappa}_{N} \varphi^{2} \sum_{\mu=1}^{4} \cos \left(k_{\mu}\right)+\frac{16 \rho^{2}}{\tilde{y}_{N}^{2} \varphi^{2}} \cdot q(k)\right) \cdot e^{i k n_{1}}
$$

where $q(k)$ denotes the eigenvalues of the matrix $|\Gamma(\Delta n)|^{2}$ given by

$$
\mathbb{R} \ni q(k)=\frac{1}{L^{4}} \sum_{p \in \mathcal{P}} \alpha^{+}(p) \cdot \alpha^{+}(\wp) \cdot \frac{\tilde{p} \cdot \tilde{\wp}}{\sqrt{\tilde{p}^{2}} \cdot \sqrt{\tilde{\wp}^{2}}}, \quad \wp=k-p .
$$

For the numerical evaluation of this quantity it is useful to use some symmetries of $q(k)$. One has $q(k)=q\left(k^{\prime}\right)$ at least, if $k^{\prime}$ is a permutation of the components of $k$, or if $k_{\mu}^{\prime}= \pm k_{\mu}$ for all $\mu$.

Now we can search for the absolute minima of the effective action in eq. (4.33). For this purpose we relate the amplitudes $m^{i}, s^{i}$ to the values of the overall magnetization $m$ and staggered magnetization $s$, respectively, according to

$$
m^{i}=\sqrt{\frac{L^{4}}{N}} m \quad \text { and } \quad s^{i}=\sqrt{\frac{L^{4}}{N}} s .
$$

With this notation one directly obtains from the effective action in eq. (4.33) the following expression in terms of the quantities $m, s$ and $\lambda$

$$
\begin{aligned}
S[m, s, \lambda]= & \frac{N}{2} \operatorname{Tr}^{\prime} \ln \left[-\kappa^{\mathrm{eff}}+\lambda\right]+\frac{N}{\tilde{t}_{N}} \cdot m^{2} \cdot L^{4} \cdot\left(-8 \tilde{\kappa}_{N} \varphi^{2}-\frac{16 \rho^{2}}{\tilde{y}_{N}^{2} \varphi^{2}} q(0)+\lambda\right) \\
& +\frac{N}{\tilde{t}_{N}} \cdot s^{2} \cdot L^{4} \cdot\left(+8 \tilde{\kappa}_{N} \varphi^{2}-\frac{16 \rho^{2}}{\tilde{y}_{N}^{2} \varphi^{2}} q(\pi)+\lambda\right)-\frac{N}{\tilde{t}_{N}} L^{4} \lambda,
\end{aligned}
$$

where the summation over the coupling matrix components has been performed by using eq. (4.35) with the settings $k=(0,0,0,0) \equiv 0$ and $k=(\pi, \pi, \pi, \pi) \equiv \pi$, respectively. 

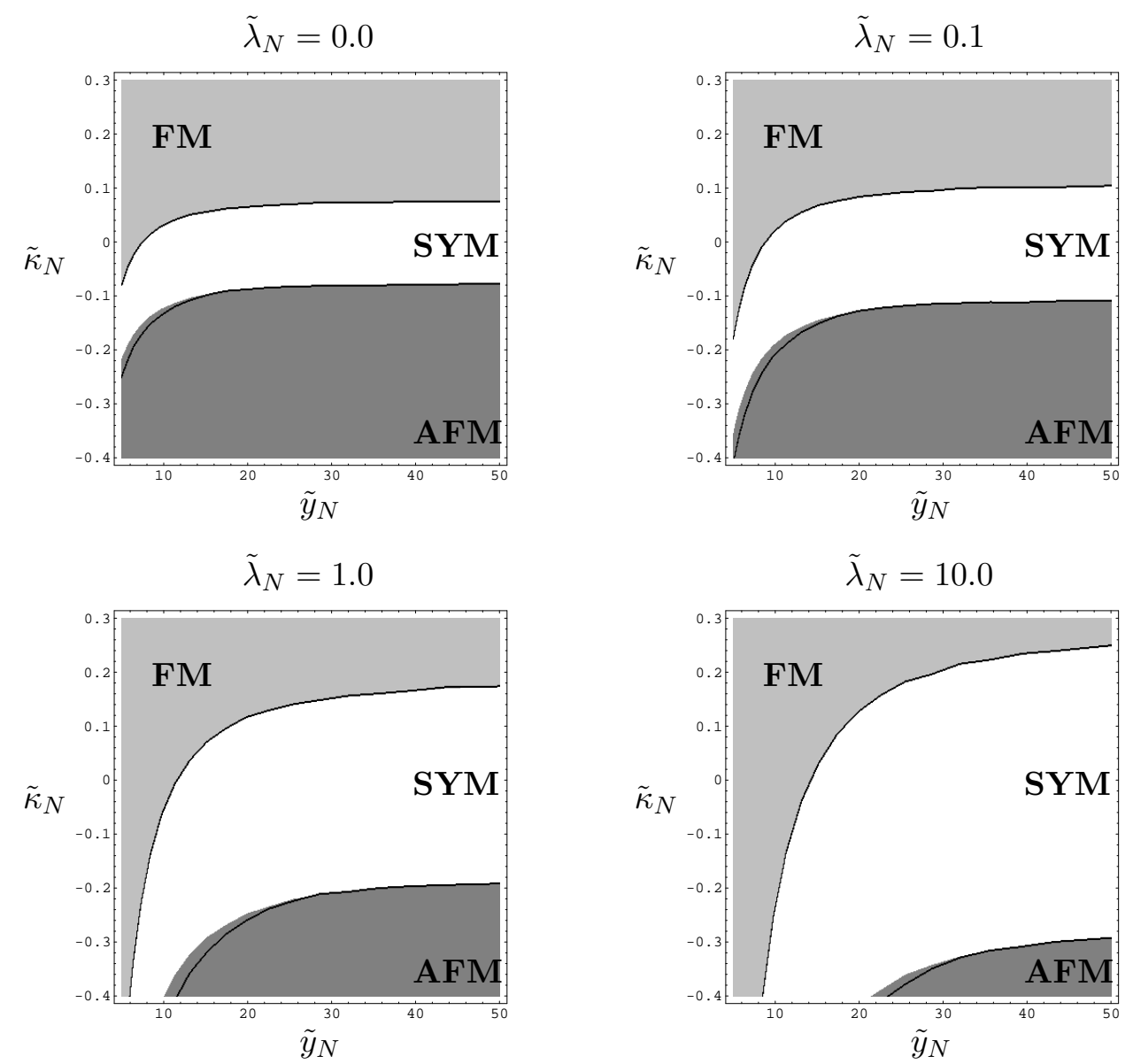

Figure 4: Phase diagrams for $L=\infty$ with respect to the Yukawa coupling constant $\tilde{y}_{N}$ and the hopping parameter $\tilde{\kappa}_{N}$ for several selected values of the quartic coupling constant $\tilde{\lambda}_{N}$. The presented phase structure was determined for $\epsilon=10^{-1}$, while the black lines show the phase transition lines obtained for $\epsilon=10^{-3}$. An explanation of the $\epsilon$-dependence of the presented results is given in the text.

Analogously to $\operatorname{det}^{\prime}, \operatorname{Tr}^{\prime}$ denotes the trace neglecting the modes $|0\rangle$ and $|\pi\rangle$. We can now derive the corresponding gap equations by differentiating with respect to $m, s$, and $\lambda$ leading to

$$
\begin{gathered}
0=m \cdot\left[\lambda-\left(8 \tilde{\kappa}_{N} \varphi^{2}+\frac{16 \rho^{2}}{\tilde{y}_{N}^{2} \varphi^{2}} \cdot q(0)\right)\right], \\
0=s \cdot\left[\lambda-\left(-8 \tilde{\kappa}_{N} \varphi^{2}+\frac{16 \rho^{2}}{\tilde{y}_{N}^{2} \varphi^{2}} \cdot q(\pi)\right)\right], \\
m^{2}+s^{2}=1-\frac{\tilde{t}_{N}}{4} \frac{1}{L^{4}} \sum_{\substack{k \in \mathcal{P} \\
0 \neq k \neq \pi}}\left[-\tilde{\kappa}_{N} \varphi^{2} \sum_{\mu=1}^{4} \cos \left(k_{\mu}\right)-\frac{8 \rho^{2}}{\tilde{y}_{N}^{2} \varphi^{2}} q(k)+\frac{\lambda}{2}\right]^{-1} .
\end{gathered}
$$


Equation (4.39) implies that $m$ or the given argument within the square brackets has to vanish. An analogous observation can be drawn from eq. (4.40). For the investigation of the phase structure we now consider two different scenarios for the amplitudes $m$ and $s$, namely a ferromagnetic phase $(m \neq 0, s=0)$ and an anti-ferromagnetic phase $(m=0$, $s \neq 0)$. For each of these cases we can then derive a self-consistency relation:

1. For a ferromagnetic phase $(\mathrm{FM})(m \neq 0, s=0)$ one obtains from 4.39)

$$
\lambda=8 \tilde{\kappa}_{N} \varphi^{2}+\frac{16 \rho^{2}}{\tilde{y}_{N}^{2} \varphi^{2}} \cdot q(0)
$$

and hence the following self-consistency relation

$$
0<m^{2}=1-\frac{\tilde{t}_{N}}{4} \frac{1}{L^{4}} \sum_{\substack{k \in \mathcal{P}_{m}(\epsilon) \\ 0 \neq k \neq \pi}}[\underbrace{\tilde{\kappa}_{N} \varphi^{2} \sum_{\mu=1}^{4}\left(1-\cos \left(k_{\mu}\right)\right)+\frac{8 \rho^{2}}{\tilde{y}_{N}^{2} \varphi^{2}}(q(0)-q(k))}_{\mathcal{W}_{m}(k)}]^{-1} .
$$

2. For an anti-ferromagnetic phase $(\mathrm{AFM})(m=0, s \neq 0)$ one obtains from $(4.40)$

$$
\lambda=-8 \tilde{\kappa}_{N} \varphi^{2}+\frac{16 \rho^{2}}{\tilde{y}_{N}^{2} \varphi^{2}} \cdot q(\pi)
$$

and hence the self-consistency relation

$$
0<s^{2}=1-\frac{\tilde{t}_{N}}{4} \frac{1}{L^{4}} \sum_{\substack{k \in \mathcal{P}_{s}(\epsilon) \\ 0 \neq k \neq \pi}}[\underbrace{-\tilde{\kappa}_{N} \varphi^{2} \sum_{\mu=1}^{4}\left(1+\cos \left(k_{\mu}\right)\right)+\frac{8 \rho^{2}}{\tilde{y}_{N}^{2} \varphi^{2}}(q(\pi)-q(k))}_{\mathcal{W}_{s}(k)}]^{-1} .
$$

Three further remarks shall be given here.

(i) The equations (4.43) and (4.45) are denoted as self-consistency relations because the assumption of a (anti-)ferromagnetic phase becomes inconsistent, if the resulting value for $m^{2}$ (or $s^{2}$, respectively) becomes non-positive. If both assumptions become inconsistent simultaneously, this corresponds to a symmetric phase (SYM) with $m=$ $s=0$, while the case $m^{2}>0$ and $s^{2}>0$ is denoted as a ferrimagnetic phase (FI).

(ii) For the ferromagnetic phase the choice of $\lambda$ according to eq. (4.42) justifies the integration performed in eq. (4.33) a posteriori, because it sufficiently shifts the eigenvalues $2 \mathcal{W}_{m}(k)$ of the matrix $-\kappa^{\mathrm{eff}}+\lambda$ to make all of them positive, except for the constant mode $(k=0)$ which was excluded from the Gauss-integration.

(iii) For the anti-ferromagnetic phase, in contrast, choosing $\lambda$ according to eq. (4.44) does not guarantee all eigenvalues $2 \mathcal{W}_{s}(k)$ of $-\kappa^{\text {eff }}+\lambda$ to be positive. The Gaussintegration in eq. (4.33) can therefore only be performed for all those modes $0 \neq k \neq \pi$ 


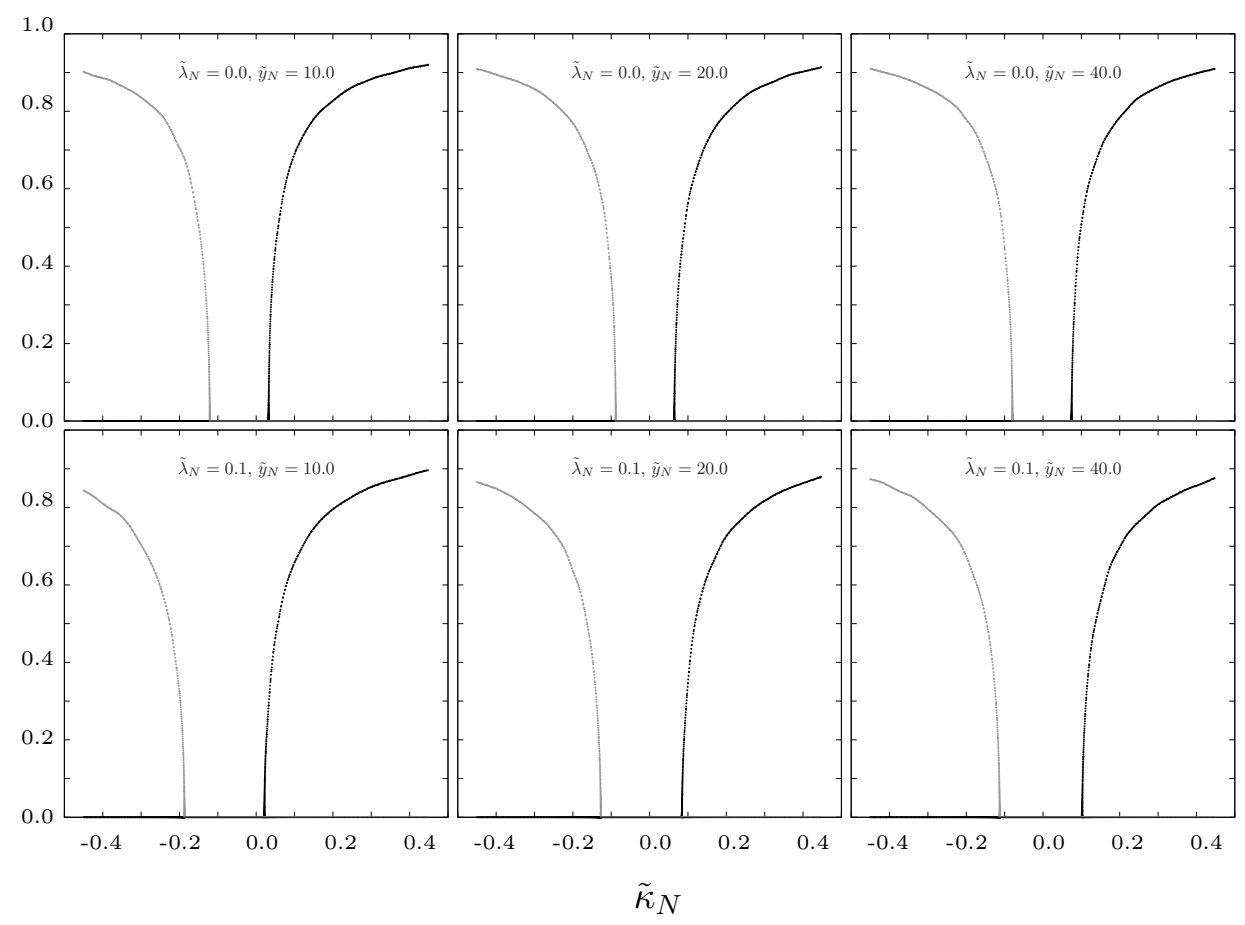

Figure 5: Expectation values for the amplitudes of the constant ( $m$ : black curve) and staggered ( $s$ : gray curve) modes for several selected values of the Yukawa coupling constant $\tilde{y}_{N}$ and the quartic coupling parameters $\tilde{\lambda}_{N}=0.0$ and $\tilde{\lambda}_{N}=0.1$. The results were obtained for $L=\infty$.

which fulfill $\mathcal{W}_{s}(k) \geq \epsilon$ with an arbitrary lower bound $\epsilon>0$. The details of this statement are presented in appendix $\mathrm{B}$. The results of this more careful consideration are already presented in eq. (4.43) and eq. (4.45). The only difference to the naive result is that the set over which the sum has to be performed is reduced from $\mathcal{P}$ to $\mathcal{P}_{s}(\epsilon)$ with the definitions

$$
\mathcal{P}_{m}(\epsilon)=\left\{k \in \mathcal{P}: \mathcal{W}_{m}(k) \geq \epsilon\right\} \text { and } \mathcal{P}_{s}(\epsilon)=\left\{k \in \mathcal{P}: \mathcal{W}_{s}(k) \geq \epsilon\right\}
$$

where the introduction of the set $\mathcal{P}_{m}(\epsilon)$ is actually unnecessary due to the previous remark (ii).

The corresponding phase structure can now be obtained by numerically evaluating equations (4.43) and (4.45). For some selected values of the quartic coupling $\tilde{\lambda}_{N}$ the resulting phase diagrams with respect to the parameters $\tilde{\kappa}_{N}$ and $\tilde{y}_{N}$ are shown in figure 4 . All presented results were obtained for an infinite lattice, i.e. $L=\infty$. For $\tilde{y}_{N} \rightarrow \infty$ the effective coupling matrix in eq. (4.30) converges to the coupling structure of a pure nearestneighbour sigma-model. One therefore expects a symmetric phase centered around $\tilde{\kappa}_{N}=0$ at large values of the Yukawa coupling constant $\tilde{y}_{N}$ as can be observed in the plots. For decreasing $\tilde{y}_{N}$ the symmetric phase bends towards negative values of $\tilde{\kappa}_{N}$. In the plots the results for the phase transition lines obtained for $\epsilon=10^{-1}$ and $\epsilon=10^{-3}$ are compared to each other. While the phase transition line to the ferromagnetic phase is unaffected by 


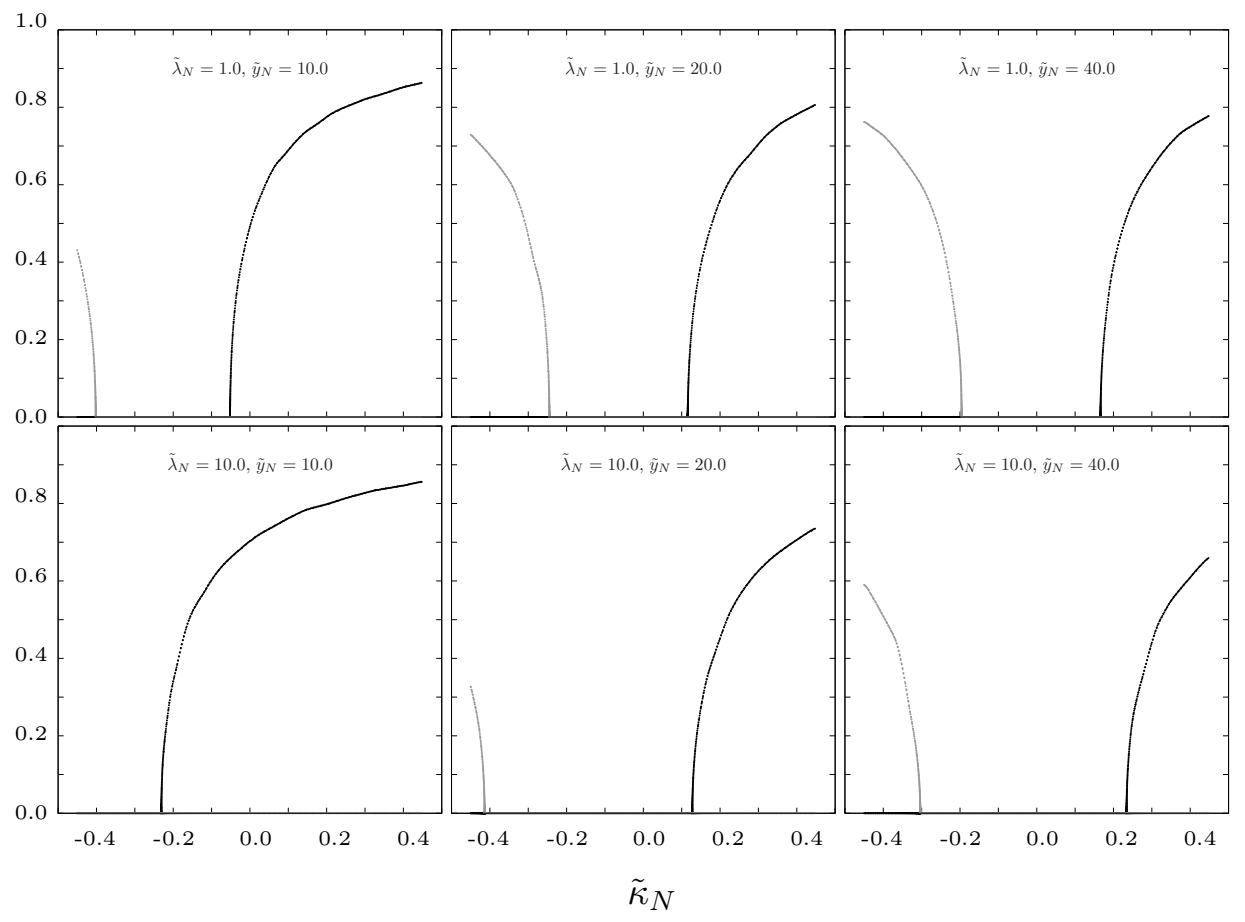

Figure 6: Expectation values for the amplitudes of the constant ( $m$ : black curve) and staggered ( $s$ : gray curve) modes for several selected values of the Yukawa coupling constant $\tilde{y}_{N}$ and the quartic coupling parameters $\tilde{\lambda}_{N}=1.0$ and $\tilde{\lambda}_{N}=10.0$. The results were obtained for $L=\infty$.

sending $\epsilon$ to zero as expected, the curves start to differ for the anti-ferromagnetic phase transition at small values of $\tilde{y}_{N}$. The discrepancy between these two lines can serve as an indicator down to which value of $\tilde{y}_{N}$ the neglection of the modes with $\mathcal{W}_{s}(k)<\epsilon$ can be considered as a good approximation (besides the uncertainties arising from cutting off the power series in eq. $(4.9)$ at small values of $\left.\tilde{y}_{N}\right)$. We add here, that we chose the presented parameter range in all phase diagrams such that the volume of the space of the considered modes is at least $95 \%$ of the volume of the whole mode space, i.e. $\operatorname{Vol}\left(\mathcal{P}_{s}(\epsilon)\right) \geq 0.95 \cdot \operatorname{Vol}(\mathcal{P})$. For $\tilde{y}_{N} \rightarrow \infty$ the volume of the neglected modes vanishes and the problem encountered during the Gauss-integration in eq. (4.33) eventually disappears.

The order of the phase transitions can again be determined by calculating the expectation values of the amplitudes of the constant and staggered modes $m$ and $s$ directly from equations (4.43) and (4.45). The corresponding results are presented in figure 5 and figure 6. One clearly sees that the occurring phase transitions are of second order as one would also expect from the limit $\tilde{y}_{N} \rightarrow \infty$ where the model becomes a sigma-model.

\section{Summary and conclusions}

In this paper we have studied analytically the phase structure of a chirally invariant lattice 
Higgs-Yukawa model, originally proposed by Lüscher. This was possible in the large $N_{f^{-}}$ limit for small as well as for large values of the Yukawa coupling constant and it could be shown that the model possesses a rich phase structure. Here we remark again that although we are eventually interested in the physical setting $N_{f}=1$ we expect the large $N_{f}$ phase diagram to be the same on a qualitative level. Thus, the large $N_{f}$ limit can provide valuable information for future calculations also for $N_{f}=1$.

In section 3 we began by considering the model at small values of the Yukawa and quartic coupling constant and argued that taking only the constant $(m)$ and staggered $(s)$ modes of the Higgs field into account is sufficient for the determination of the phases in that regime of the Yukawa and quartic coupling constant. We then presented an explicit expression for the effective potential at tree-level in terms of $m$ and $s$ and showed the corresponding phase diagrams for some selected values of the quartic coupling constant. In these diagrams all possible phases, i.e. symmetric $(m=0, s=0)$, ferromagnetic $(m \neq 0$, $s=0)$, anti-ferromagnetic $(m=0, s \neq 0)$, and ferrimagnetic phases $(m \neq 0, s \neq 0)$, could be observed. Furthermore, we concluded from our result for the effective potential that the occurring phase transitions from the symmetric to the ferromagnetic and anti-ferromagnetic phases are of second order.

In the following section 4 we proceeded to the regime of large values of the Yukawa coupling constant $y_{N}$. We showed that for sufficiently large values of $y_{N}$ and arbitrary values of the quartic coupling constant $\lambda_{N}$ the model becomes an $O(4)$-symmetric, nonlinear sigma-model in the large $N_{f}$-limit up to some finite-volume terms. In particular, this relation to a sigma-model has the consequence that a symmetric phase also exists at large values of the Yukawa coupling constant. We determined the phase structure of the latter sigma-model by an additional large $N$-limit with $N$ denoting the number of Higgs field components here. The corresponding phase diagrams revealed again a rich structure consisting of symmetric, ferromagnetic, and anti-ferromagnetic phases separated by second order phase transitions. The symmetric phase, however, was shown to emerge only in the infinite volume limit. For small lattices, finite volume effects cause an asymmetry in $m$ and $s$ which one would not expect in a pure sigma-model. These finite volume effects may easily give rise to a misleading interpretation that a symmetric phase at strong values of the Yukawa coupling constant does not exist. However, on sufficiently large lattices the symmetric phase should become clearly observable and the asymmetry should disappear.

The validity of our analytical results and in particular the latter predictions about the symmetric phase at large $y_{N}$ have been confronted in ref. [16] with the results of corresponding Monte-Carlo simulations including the chiral invariant fermions in a fully dynamical manner.

\section{A. Details of calculation I}

In this appendix we would like to make up for the neglected derivation of eq. (4.14). 
Starting from eq. (4.11) one finds

$$
\begin{aligned}
\operatorname{Tr}[ & \left.\mathcal{A} B^{-1}\right]^{r}=\sum_{n_{1}, \ldots, n_{r}} \operatorname{Tr}_{8 \times 8}\left(\left[\mathcal{A} B^{-1}\right]_{n_{1}, n_{2}} \cdot \ldots \cdot\left[\mathcal{A} B^{-1}\right]_{n_{r}, n_{1}}\right) \\
= & \sum_{n_{1}, \ldots, n_{r}} \sum_{\substack{\zeta_{1} \epsilon_{1} k_{1}, \ldots, \zeta_{r} \epsilon_{r} k_{r} \\
\zeta_{1}^{\prime} \epsilon_{1}^{\prime} k_{1}^{\prime}, \ldots, \zeta_{r}^{\prime} \epsilon_{r}^{\prime} k_{r}^{\prime}}} \sum_{p_{1}, \ldots, p_{r} \in \mathcal{P}} \frac{e^{i p_{1}\left(n_{1}-n_{2}\right)}}{L^{4}} \cdot \ldots \cdot \frac{e^{i p_{r}\left(n_{r}-n_{1}\right)}}{L^{4}} \\
& \times \operatorname{Tr}_{8 \times 8}[u^{\zeta_{1} \epsilon_{1} k_{1}}\left(p_{1}\right) \alpha^{\epsilon_{1}}\left(p_{1}\right)\left(\hat{B}^{\left(p_{1}\right)}\left(\Phi_{n_{2}}^{*} /\left|\Phi_{n_{2}}\right|^{2}\right)\right)_{\zeta_{1} \epsilon_{1} k_{1}, \zeta_{1}^{\prime} \epsilon_{1}^{\prime} k_{1}^{\prime}} \underbrace{\left[u^{\zeta_{1}^{\prime} \epsilon_{1}^{\prime} k_{1}^{\prime}}\left(p_{1}\right)\right]^{\dagger} \cdot u^{\zeta_{2} \epsilon_{2} k_{2}}\left(p_{2}\right)}_{U\left(p_{1}, p_{2}\right)_{\zeta_{1}^{\prime} \epsilon_{1}^{\prime} k_{1}^{\prime}, \zeta_{2} \epsilon_{2} k_{2}}} \\
& \times \alpha^{\epsilon_{2}}\left(p_{2}\right)\left(\hat{B}^{\left(p_{2}\right)}\left(\Phi_{n_{3}}^{*} /\left|\Phi_{n_{3}}\right|^{2}\right)\right)_{\zeta_{2} \epsilon_{2} k_{2}, \zeta_{2}^{\prime} \epsilon_{2}^{\prime} k_{2}^{\prime}}\left[u^{\zeta_{2}^{\prime} \epsilon_{2}^{\prime} k_{2}^{\prime}}\left(p_{2}\right)\right]^{\dagger} \cdot \ldots \cdot u^{\zeta_{r} \epsilon_{r} k_{r}}\left(p_{r}\right) \alpha^{\epsilon_{r}}\left(p_{r}\right) \\
& \left.\times\left(\hat{B}^{\left(p_{r}\right)}\left(\Phi_{n_{1}}^{*} /\left|\Phi_{n_{1}}\right|^{2}\right)\right)_{\zeta_{r} \epsilon_{r} k_{r}, \zeta_{r}^{\prime} \epsilon_{r}^{\prime} k_{r}^{\prime}}\left[u^{\zeta_{r}^{\prime} \epsilon_{r}^{\prime} k_{r}^{\prime}}\left(p_{r}\right)\right]^{\dagger}\right] \\
= & \sum_{n_{1}, \ldots, n_{r}} \sum_{p_{1}, \ldots, p_{r} \in \mathcal{P}} \operatorname{Tr}_{8 \times 8}\left[\prod_{i=1}^{r} \frac{e^{i p_{i}\left(n_{i}-n_{i+1}\right)}}{L^{4}}\left|\Phi_{n_{i+1}}\right|^{-2} \mathcal{A}\left(p_{i}\right)\left(\hat{B}^{\left(p_{i}\right)}\left(\Phi_{n_{i+1}}^{*}\right)\right) U\left(p_{i}, p_{i+1}\right)\right],
\end{aligned}
$$

where the definition of the spinor basis transformation matrix $U\left(p_{1}, p_{2}\right)$ given in eq. (3.4) was used.

\section{B. Details of calculation II}

In this appendix we want to deal with the possibly non-positive eigenvalues of the operator $-\kappa^{\text {eff }}+\lambda$, which would not allow the option of performing the Gauss-integration in eq. (4.33) over all modes, in a more precise manner. We therefore restart our calculation beginning in eq. (4.31). Now we perform the Gauss-integration solely over those modes $k \in \mathcal{P}, 0 \neq k \neq \pi$ which have their corresponding eigenvalue of the operator $-\kappa^{\text {eff }}+\lambda$ not smaller than $2 \epsilon>0$. We denote the subset of these modes as $\mathcal{P}(\epsilon, \lambda)$. According to eq. 4.35) it is given as

$$
\mathcal{P}(\epsilon, \lambda)=\left\{k \in \mathcal{P}:-\tilde{\kappa}_{N} \varphi^{2} \sum_{\mu=1}^{4} \cos \left(k_{\mu}\right)-\frac{8 \rho^{2}}{\tilde{y}_{N}^{2} \varphi^{2}} \cdot q(k)+\frac{\lambda}{2} \geq \epsilon\right\} .
$$

Performing the Gauss-integration only over these modes the action reduces to

$$
\begin{aligned}
& S\left[m^{i}, s^{i}, \lambda, \sigma_{k}^{i}\right]=-\ln \left[\operatorname{det}^{\prime \prime}\left(-\kappa^{\mathrm{eff}}+\lambda\right)\right]^{-N / 2}+\frac{1}{t_{N}}\left\{\sum_{i=1}^{N}\left[m^{i}\right]^{2} \cdot\left\langle 0\left|-\kappa^{\mathrm{eff}}+\lambda\right| 0\right\rangle-L^{4} \lambda\right. \\
& \left.+\sum_{i=1}^{N}\left[s^{i}\right]^{2} \cdot\left\langle\pi\left|-\kappa^{\mathrm{eff}}+\lambda\right| \pi\right\rangle+\sum_{i=1}^{N} \sum_{\substack{k \in \mathcal{P}(\epsilon, \lambda) \\
0 \neq k \neq \pi}}\left[\sigma_{k}^{i}\right]^{2} \cdot\left\langle k\left|-\kappa^{\mathrm{eff}}+\lambda\right| k\right\rangle\right\} \\
& =\frac{N}{2} \operatorname{Tr}^{\prime \prime} \ln \left[-\kappa^{\mathrm{eff}}+\lambda\right]+\frac{N}{\tilde{t}_{N}} \cdot m^{2} \cdot L^{4} \cdot\left(-8 \tilde{\kappa}_{N} \varphi^{2}-\frac{16 \rho^{2}}{\tilde{y}_{N}^{2} \varphi^{2}} q(0)+\lambda\right) \\
& +\frac{N}{\tilde{t}_{N}} \cdot s^{2} \cdot L^{4} \cdot\left(+8 \tilde{\kappa}_{N} \varphi^{2}-\frac{16 \rho^{2}}{\tilde{y}_{N}^{2} \varphi^{2}} q(\pi)+\lambda\right)-\frac{N}{\tilde{t}_{N}} L^{4} \lambda \\
& +\sum_{\substack{k \in \tilde{\mathcal{P}}_{(\epsilon, \lambda)} \\
0 \neq k \neq \pi}} \frac{N}{\tilde{t}_{N}} \cdot \sigma_{k}^{2} \cdot L^{4} \cdot\left(-2 \tilde{\kappa}_{N} \varphi^{2} \sum_{\mu=1}^{4} \cos \left(k_{\mu}\right)-\frac{16 \rho^{2}}{\tilde{y}_{N}^{2} \varphi^{2}} q(k)+\lambda\right)
\end{aligned}
$$


where $\sigma_{k}^{i}$ denote the amplitudes of the excluded modes with $k \in \overline{\mathcal{P}}(\epsilon, \lambda), 0 \neq k \neq \pi$ and $\overline{\mathcal{P}}(\epsilon, \lambda) \equiv \mathcal{P} / \mathcal{P}(\epsilon, \lambda)$ is the complement of $\mathcal{P}(\epsilon, \lambda)$. Here the notation

$$
\sigma_{k}^{i}=\sqrt{\frac{L^{4}}{N}} \sigma_{k}
$$

was introduced correspondingly to eq. (4.37) and the plane wave modes $|k\rangle$ were explicitly given in eq. (4.34). The determinant $\operatorname{det}^{\prime \prime}$ and the trace $\operatorname{Tr}^{\prime \prime}$, respectively, are now only performed over the modes $k \in \mathcal{P}(\epsilon, \lambda), 0 \neq k \neq \pi$, as desired. The resulting gap equations can now be obtained by differentiating the effective action with respect to $m, s, \lambda$ and all $\sigma_{k}$. This leads again to eq. (4.39) and eq. (4.40). Only the third one, eq. (4.41), is modified yielding now

$$
m^{2}+s^{2}+\sum_{\substack{k \in \mathcal{P}_{(}(\epsilon, \lambda) \\ 0 \neq k \neq \pi}} \sigma_{k}^{2}=1-\frac{\tilde{t}_{N}}{4} \frac{1}{L^{4}} \sum_{\substack{k \in \mathcal{P}(\epsilon, \lambda) \\ 0 \neq k \neq \pi}}\left[-\tilde{\kappa}_{N} \varphi^{2} \sum_{\mu=1}^{4} \cos \left(k_{\mu}\right)-\frac{8 \rho^{2}}{\tilde{y}_{N}^{2} \varphi^{2}} q(k)+\frac{\lambda}{2}\right]^{-1}
$$

Furthermore, one obtains one additional gap equation for every mode $k \in \overline{\mathcal{P}}(\epsilon, \lambda), 0 \neq k \neq$ $\pi$ according to

$$
0=\sigma_{k} \cdot\left[\lambda-\left(+2 \tilde{\kappa}_{N} \varphi^{2} \sum_{\mu=1}^{4} \cos \left(k_{\mu}\right)+\frac{16 \rho^{2}}{\tilde{y}_{N}^{2} \varphi^{2}} q(k)\right)\right] \quad \forall k \in \overline{\mathcal{P}}(\epsilon, \lambda), 0 \neq k \neq \pi .
$$

Again we consider the scenario of a purely ferromagnetic phase and the scenario of a purely anti-ferromagnetic phase for the investigation of the phase structure. The only particularity here is that we assume all $\sigma_{k}$ to be zero in both cases. (In principle, with this approach one could also study the phase structure of some of the amplitudes $\sigma_{k}$, but this is beyond our interest here.) We thus arrive directly at the prior equations (4.42) and (4.44), respectively, fixing the value of $\lambda$ as before. With this fixation of $\lambda$ the subset $\mathcal{P}(\epsilon, \lambda)$ now becomes $\mathcal{P}_{m}(\epsilon)$ for the ferromagnetic phase as already defined in eq. (4.46). For the anti-ferromagnetic phase it becomes $\mathcal{P}_{s}(\epsilon)$. We have now arrived at the final results for the self-consistency equations that were already presented in eq. (4.43) and eq. (4.45).

In order to get a rough estimate about the validity of neglecting the modes $k \in \overline{\mathcal{P}}(\epsilon, \lambda)$ one should check the volume of this subset and compare it to the volume of the full set $\mathcal{P}$ as we did in our discussion in the main text.

\section{Acknowledgments}

We thank the "Deutsche Telekom Stiftung" for supporting this study by providing a Ph.D. scholarship for P.G. We further acknowledge the support of the DFG through the DFGproject Mu932/4-1. Furthermore, we are grateful to Joel Giedt, Michael Müller-Preussker, Erich Poppitz, and Gian Carlo Rossi for helpful discussions and comments. 


\section{References}

[1] J. Smit, Standard model and chiral gauge theories on the lattice, Nucl. Phys. 17 (Proc. Suppl.) (1990) 3.

[2] J. Shigemitsu, Higgs-Yukawa chiral models, Nucl. Phys. 20 (Proc. Suppl.) (1991) 515.

[3] M.F.L. Golterman, Lattice chiral gauge theories: results and problems, Nucl. Phys. 20 (Proc. Suppl.) (1991) 528 .

[4] I. Montvay and G. Münster. Quantum Fields on a lattice, (Cambridge Monographs on Mathematical Physics), Cambridge University Press (1997).

[5] A.K. De and J. Jersák. Yukawa models on the lattice, Jülich, HLRZ 91-83, preprint edition (1991).

[6] M.F.L. Golterman, D.N. Petcher and E. Rivas, On the Eichten-Preskill proposal for lattice chiral gauge theories, Nucl. Phys. 29BC (Proc. Suppl.) (1992) 193 hep-lat/9207005.

[7] K. Jansen, Domain wall fermions and chiral gauge theories, Phys. Rept. 273 (1996) 1 hep-lat/9410018.

[8] M. Lüscher, Exact chiral symmetry on the lattice and the Ginsparg-Wilson relation, Phys. Lett. B 428 (1998) 342 hep-lat/9802011.

[9] H. Neuberger, More about exactly massless quarks on the lattice, Phys. Lett. B 427 (1998) 353 hep-lat/9801031.

[10] H.B. Nielsen and M. Ninomiya, Absence of neutrinos on a lattice. 1 Proof by homotopy theory, Nucl. Phys. B 185 (1981) 20 [Erratum ibid. B195 (1982) 541].

[11] A. Hasenfratz, P. Hasenfratz, K. Jansen, J. Kuti and Y. Shen, The equivalence of the top quark condensate and the elementary Higgs field, Nucl. Phys. B 365 (1991) 79.

[12] A. Hasenfratz, K. Jansen and Y. Shen, The phase diagram of a U(1) Higgs-Yukawa model at finite lambda, Nucl. Phys. B 394 (1993) 527 hep-lat/9207006.

[13] P. Gerhold and K. Jansen, On the phase structure of a chiral invariant Higgs-Yukawa model PoS(LAT2006) 043.

[14] J. Giedt and E. Poppitz, Chiral lattice gauge theories and the strong coupling dynamics of a Yukawa-Higgs model with Ginsparg-Wilson fermions, hep-lat/0701004.

[15] J. Giedt and E. Poppitz, private communication (2007).

[16] P. Gerhold and K. Jansen, The phase structure of a chirally invariant lattice Higgs-Yukawa model - numerical simulations, arXiv:0707.3849.

[17] P. Hernandez, K. Jansen and M. Lüscher, Locality properties of Neuberger's lattice Dirac operator, Nucl. Phys. B 552 (1999) 363 hep-lat/9808010.

[18] H. Flyvbjerg, 1/N expansion of the nonlinear $\sigma$-model, Phys. Lett. B 219 (1989) 323.

[19] J. Zinn-Justin. Quantum field theory and critical phenomena, Oxford University Press, 2nd edition (1993). 